This article was downloaded by: [Univ Du Quebec A Chicoutimi]

On: 09 January 2014, At: 11:21

Publisher: Routledge

Informa Ltd Registered in England and Wales Registered Number: 1072954 Registered

office: Mortimer House, 37-41 Mortimer Street, London W1T 3J H, UK

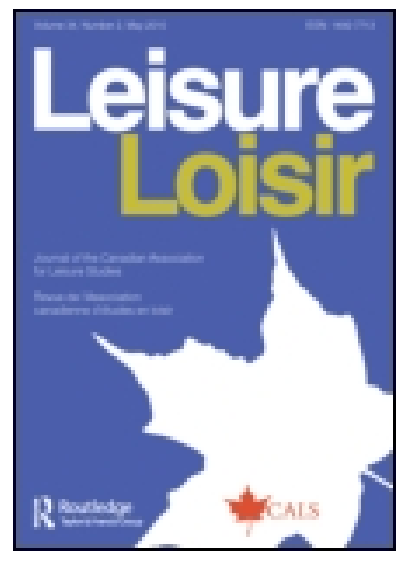

\title{
Leisure/Loisir
}

Publication details, including instructions for authors and subscription information:

http:// www. tandfonline.com/ loi/ rloi20

\section{Questionnement prospectif à partir de quelques tendances sociales et de leurs impacts sur le loisir}

\author{
Gervais Deschênes ${ }^{a}$ \\ a Université du Québec à Chicoutimi, 555 Boulevard Université, \\ Chicoutimi, Québec G7H 2B1, Canada \\ Published online: 09 Oct 2013.
}

To cite this article: Gervais Deschênes (2013) Questionnement prospectif à partir de quelques tendances sociales et de leurs impacts sur le loisir, Leisure/ Loisir, 37:3, 205-231, DOI:

10.1080/14927713.2013.842729

To link to this article: http:// dx. doi.org/ 10.1080/ 14927713.2013.842729

\section{PLEASE SCROLL DOWN FOR ARTICLE}

Taylor \& Francis makes every effort to ensure the accuracy of all the information (the "Content") contained in the publications on our platform. However, Taylor \& Francis, our agents, and our licensors make no representations or warranties whatsoever as to the accuracy, completeness, or suitability for any purpose of the Content. Any opinions and views expressed in this publication are the opinions and views of the authors, and are not the views of or endorsed by Taylor \& Francis. The accuracy of the Content should not be relied upon and should be independently verified with primary sources of information. Taylor and Francis shall not be liable for any losses, actions, claims, proceedings, demands, costs, expenses, damages, and other liabilities whatsoever or howsoever caused arising directly or indirectly in connection with, in relation to or arising out of the use of the Content.

This article may be used for research, teaching, and private study purposes. Any substantial or systematic reproduction, redistribution, reselling, loan, sub-licensing, systematic supply, or distribution in any form to anyone is expressly forbidden. Terms \& Conditions of access and use can be found at http://www.tandfonline.com/page/termsand-conditions 


\title{
Questionnement prospectif à partir de quelques tendances sociales et de leurs impacts sur le loisir
}

\author{
Gervais Deschênes* \\ Université du Québec à Chicoutimi, 555 Boulevard Université, Chicoutimi, Québec G7H 2B1, \\ Canada
}

(Received February 2013; final version received August 2013)

In continuing prospective discussions on ethical and spiritual aspects of leisure, we would like to identify in this paper a broader horizon of possibilities which answer the question asked by the ancient philosophers: What is the worth of practicing leisure? Or, to be more up to date: Leisure experiences! Yes of course, but what for? In developing this vision of leisure, the identification of significant social trends is crucial because it indicates a risk that may have a gain or a loss if we do not act now for the better. To identify social trends, we start from the thinking of authors in various disciplines, proceeding by successive waves from the perspective of the social construction of reality, where it is possible afterwards to identify and talk about certain ethical and spiritual aspects of leisure which benefit the world's citizens.

Keywords: prospective approach; voluntary action; therapeutic leisure; leisure education; spirituality of leisure

Dans la poursuite d'un questionnement prospectif sur les enjeux du loisir, nous voudrions dégager un horizon plus élargi de possibilités qui répond à la question posée par les philosophes anciens: Qu'est-ce que cela vaut-il de pratiquer le loisir? Ou pour être mieux de son époque: Des loisirs! Oui je veux bien, mais pour quoi faire? Pour élaborer notre vision du loisir, l'identification de quelques tendances sociales significatives est primordiale parce qu'elle révèle un risque pouvant comporter une perte ou un gain à contenir si nous n'agissons pas maintenant pour le meilleur. Pour identifier les tendances sociales, nous partirons de la pensée d'auteurs de champs disciplinaires divers en procédant par vagues successives dans une perspective de construction sociale de la réalité, où il est possible après coup d'identifier et de discourir amplement sur certains enjeux du loisir au bénéfice des citoyen(ne)s du monde.

Mots-clés: approche prospective; action bénévole; loisir thérapeutique; éducation au loisir et spiritualité du loisir

\author{
L'homme de l'avenir vaudra \\ ce que vaudront ses loisirs. \\ — Jean Guéhenno (1890-1978) \\ Ce que je crois
}

Il faut faire le pari que les avancées du bien se cumulent, mais que les interruptions du mal ne font pas système.

— Paul Ricoeur (1913-2005) 


\section{Quelques réflexions de départ en guise d'introduction}

Dans une civilisation qui lutte âprement face à la crise économique et à l'accroissement du chômage, la vision de la société du loisir ${ }^{1}$ pour le plus grand nombre telle qu'avancée au début des années 60 (Dumazedier, 1962), où les personnes profiteraient d'un véritable temps de loisir disponible à soi, à la condition que les moyens financiers soient suffisants, ne s'est décidément pas matérialisée. Cette vision s'est plutôt fragmentée en multiples formes parcellaires d'activités des loisirs tributaires à une paix sociale relative à sauvegarder et par l'émergence des loisirs de type marchandise que l'on retrouve dans les événements sportifs, les festivals musicaux et populaires, les spectacles d'humour, les pièces de théâtre, les activités de plein air, le tourisme ainsi que la libre circulation d'objets hétéroclites de [sur]consommation (c.-à-d. jouets de tous genres, jeux de société, gadgets, disques DVD vidéo et cédéroms de musique et chanson, ordinateurs portables, iPad, cellulaires, etc.). Ces loisirs subsidiaires deviennent qu'une simple récompense de la contribution à l'effort de production du système étatique et économique. Elles sont même considérées comme un instrument de contrôle social, où les personnes sont emprisonnées dans une 'cage de fer' (Weber, 1964). En ce sens, plusieurs penseurs considèrent cette typologie des loisirs comme aliénée et aliénante, car ils sont utilisés par les tenants de la rectitude politique qui s'efforcent à contrôler de l'extérieur une population insouciante afin qu'elle ne puisse réfléchir aux véritables problèmes de l'existence terrestre. ${ }^{2}$ Afin de rester au pouvoir, le champ du politique cherche à faire miroiter à la population une vie de divertissement par l'utilisation outrageante d'activités de type guerrier qui font la promotion d'une idéologie castratrice. Par là, les enjeux cruciaux aux destinées humaines et collectives sont alors évacués. Pourtant, « le loisir concerne les gens et est un phénomène de la vie » (Arendt, 1972, p. 266). Il demeure un droit fondamental de la personne. ${ }^{3}$ Il s'agit de reconnaître que le loisir n'est pas un temps vide, évasif, ou encore, un temps réservé pour quelques privilégiés. Il n'est pas un temps improductif, un divertissement futile ou même un simple temps indispensable à la reconstitution de la force et de récupération du travail. En fait, le temps libre consacré au loisir est compris comme un moment de socialisation, de convivialité, de connaissance éducative en vue d'être une personne à part entière dans une société, où il fait bon d'exister et d'y vivre paisiblement.

Force est de constater que l'univers du loisir comme phénomène « est 'là' devant nous. Il 'existe', il a sa propre dynamique interne, et il commande à son tour une étude en profondeur. [. . . ] Or une telle 'existence' du loisir constitue ce qu'on peut appeler une donnée culturelle, [. . .] et, disons-le immédiatement, une valeur » (Pronovost, 1983, p. 277). À ce titre, le loisir se définit comme « une activité choisie dans une liberté relative pour ses qualités de satisfaction » (Kelly, 2012, p. 3, trad.). Il s'accomplit pour l'expérience en luimême. Il est multidimensionnel, complexe et fait partie prenante de la quête identitaire des personnes dans sa liberté d'être et de devenir (Kelly, 1987). Le loisir représente un « réenchantement du monde » (Dumazedier, 1993, p. 378). À ce propos, force est de souligner qu'il n'est pas uniquement une affaire de gestion administrative, mercantile ou de prestige, mais qu'il est avant tout une pratique humaine à respecter pleinement dans son indéterminisme et sa créativité qui donne un sens de la vie à la fois personnel et social aussi longtemps que cela le permet (Bellefleur, 1975-1976, 2002). Cela dit, le loisir subit des transformations qui vont parfois à l'encontre de la croissance humaine et réclame ici et là un ajustement pour donner un sens de la vie. Il s'adapte aux situations sociales telles que l'urbanisation, l'économie, le multiculturalisme, le rapport entre les sexes, l'expansion géographique, les nouvelles technologiques, la révolution sexuelle, les mutations familiales, les communications, les conflits de valeurs ainsi que l'éducation 
(Kelly \& Freysinger, 2000). Dans ce questionnement prospectif, nous voudrions dégager un horizon plus élargi de possibilités pour répondre à la question posée antérieurement par les philosophes anciens : Qu'est-ce que cela vaut-il de pratiquer le loisir? Ou pour être de son temps : Des loisirs! Oui je veux bien, mais pour quoi faire?

\section{Méthode suivie}

Ce questionnement prospectif se réfère à la sociologie du loisir qui cherche à partir de questions de société à « soulever des débats de fond sur l'orientation même des sociétés contemporaines et tenter d'illustrer comment les études du loisir peuvent contribuer à une meilleure connaissance des enjeux qui les sous-tendent » (D’Amours \& Pronovost, 1990, p. 32). Sans tomber dans les méandres des statistiques de type quantitatif qui mènent le plus souvent à nulle part, il est pertinent d'observer et de saisir notre présent 'tel qu'il est' (tendances sociales) afin de mieux comprendre nos interventions en prodiguant le sens de la vie dans un avenir à court et moyen terme de ce 'qui devrait être' (enjeux du loisir). Cette démarche réflexive n'est donc pas déterministe, mais procède plutôt d'un sens interne et s'inscrit dans une vision globale du monde et des interactions symboliques entre les personnes. Ainsi, « [p]our agir, comprendre, analyser et intervenir dans le monde social, le chercheur doit enrichir l'objectivation scientifique par l'entrée de son propre point de vue à son tour objectivé » (Beaupré, 2012, p. 97). Cette recherche s'enracine dans la dignité de la personne puisque « toute personne a une signification telle qu'elle ne peut être remplacée à la place qu'elle occupe dans l'univers des personnes » (Mounier, 2010, p. 61). ${ }^{4}$ À partir de là, tout devient possible pour créer son propre univers de sens du loisir que chaque personne construit spécifiquement à sa manière, c'est-à-dire un univers de symboles et de signes particuliers propre à chaque personnalité individuelle en vue du bien commun. Ce questionnement prospectif s'adresse en particulier aux preneurs de décisions et ceux qui influencent le cours des événements sociaux, dont nous sommes tous à divers degrés, témoin des tendances sociales et des enjeux du loisir. Il a le souci de nourrir la pensée bienveillante dans lequel nous nous identifions le plus. Cet exercice s'attache également à ouvrir des horizons; à poser un regard sur l'évolution du loisir et de ses formes de pratique.

Pour élaborer notre projet, l'identification des tendances sociales significatives nous semble essentielle parce qu'elles révèlent des enjeux du loisir qui comportent le risque d'une perte ou d'un gain à contenir si nous n'agissons pas pour le meilleur. Pour identifier les tendances sociales, nous partirons des travaux de recherche d'auteurs provenant de champs disciplinaires divers en procédant par vagues successives dans une perspective de construction sociale de la réalité, c'est-à-dire ce qui est facilement reconnaissable par plusieurs membres de la société comme « à la fois un produit de l'homme et une objectivation de la subjectivité humaine » (Berger \& Luckmann, 1986, p. 53). Le pragmatisme séquentiel employé est également un moyen convenable pour identifier la thématique des tendances sociales à partir du premier regard de comment les choses se passent autour de notre environnement social. Dans un second regard, il apporte une interprétation évaluative; ce sur quoi il s'agit de changer pour le meilleur en vue d'agir dans une approche réaliste et persuasive pour ne pas demeurer à ce qui ne fait aucun sens puisque « [1]e sens pratique comme maîtrise pratique du sens des pratiques et des objets permet de cumuler tout ce qui va dans le même sens, tout ce qui s'assortit au moins grossièrement tout en s'ajustant aux fins poursuivies » (Bourdieu, 1980, p. 434). Les tendances sociales identifiées permettront de mieux tabler sur les enjeux du loisir dans l'intention d'émettre des éléments de solutions vis-à-vis des impacts sociaux négatifs à résorber. 
Dans ces deux étapes distinctes de la recherche, une sérielle de questions sera postulée en laissant présager un nombre appréciable de réponses que le lecteur a le libre arbitre d'entrevoir et de choisir parce que « chaque question [détient] une force que la réponse ne [contient] plus » (Wiesel, 1958, p. 13). Le but de ce questionnement prospectif est donc de donner à penser par soi-même pour que le lecteur construise son propre univers de sens du loisir en devenant davantage responsable de cette réalité expérientielle. Autrement dit, cette démarche prospective porte à réfléchir à partir du présent plutôt qu'à déclarer des allégations tous azimuts empêchant les personnes à créer des avenues de sens et de solution. Il est alors loisible d'expliquer et de mieux comprendre les événements sociaux autour de soi en lien avec le loisir.

\section{Les tendances sociales en présence}

En prévision de mieux saisir les enjeux du loisir, voici les trois tendances sociales identifiées qui seront explorées : (1) l'accroissement du temps libre; (2) le vieillissement de la population; et (3) les valeurs qui font vivre. Mentionnons au passage que ces tendances sociales ne font pas l'objet d'un choix arbitraire, mais font plutôt partie d'une inscription de sens pragmatique déjà bien établit (Deschênes, 2007, 2011; Dumazedier, 1962, 1990a, 1993; Kelly, 1987, 2012). La quête de sens du loisir devient profitable pour chaque membre de la société par une approche spéculative pour le mieux-être des personnes de la contemporanéité.

\section{Tendance sociale \# 1 - L'accroissement du temps libre}

Le temps libre est le temps libéré du travail et de tout travail supplémentaire. Il représente le temps accordé au transport utile pour se rendre au lieu de travail ainsi que les obligations personnelles telles que les repas, l'entretien personnel, le sommeil, la sexualité, les engagements familiaux, sociaux et spirituels/religieux. Le temps libre comprend le temps consacré au loisir. Une question se pose ici : Avons-nous plus ou moins de temps libre?

Schor (1991) affirme une croissance indéniable, données statistiques à l'appui, du temps de travail. Le temps libre disponible deviendrait plus rare, et par voie de conséquence, le déclin inexorable du temps de loisir. Pourtant, d'autres théoriciens du loisir ne sont pas d'accord avec ces conclusions hâtives. Sobel (1992) critique cette recherche parce qu'elle élude la complexité démographique de l'objet d'étude. Il prétend que Schor a recours à des statistiques pour conclure à des propos anecdotiques, des généralisations et suscitent des stéréotypes sociaux.

Robinson (1991) a réalisé une enquête sur l'emploi du temps en recueillant des données précises de répondants qui ont fourni de l'information systématique sur l'utilisation de leur temps au quotidien. À partir de sondages réalisés dans les années 1960, 1970 et 1980, il fait la découverte que le temps libre à augmenté de $10 \%$ durant ces périodes. Pour plus de précision, Robinson et Godbey (2000) soutiennent que les personnes sont moins actives au travail et à la maison qu'ils ne pensent le faire effectivement, et conséquemment, ont plus de temps libre disponible qu'ils ne le réalisent vraiment. Autrement dit, le temps libre dépend de la manière dont chaque personne imagine et cultive intérieurement en elle-même l'unicité de sa temporalité.

Pronovost met également en doute les affirmations de Schor (1991). Il soutient qu'il y a « une lente croissance du temps libre » et une « réduction à long terme du temps de 
travail » (1993, p. 239). D'après ces recherches, le loisir est le grand gagnant du temps déplacé puisque les Canadien(nes) profitent d'un temps non négligeable de $6 \mathrm{~h} 15 \mathrm{~min}$ utes de loisir quotidiennement (Pronovost, 1997, p. 234). Cet auteur prétend cependant que ce sont surtout les jeunes et les personnes aînées qui profitent au mieux de ce temps discrétionnaire au loisir.

Dumazedier $(1993)^{5}$ souligne que pour la plupart des travailleurs en milieu urbain, la durée du temps de travail était en moyenne d'environ 4000 heures par an au milieu du dix-neuvième siècle (env. 75 à 80 heures/sem.) tandis que lors de la seconde moitié du vingtième siècle on assiste à la diminution du temps de travail avec 1600 heures par an (env. 35 à 40 heures/sem.) avec des soirées plus longues, des week-ends, des vacances payées, du temps libre pour une retraite à 55 ans avec une espérance de vie de plus en plus allongée. ${ }^{6}$ Cette augmentation du temps libre depuis la Révolution industrielle a permis une augmentation du temps libéré au loisir. Kelly (2012) certifie également cette augmentation du temps libre et de la diminution du temps de travail hebdomadaire et affirme que les heures allouées dans les industries aux États-Unis vont selon les données suivantes : 70 heures en 1850, 65 en 1870, 62 en 1890, 55 en 1910, 45 en 1930, 42 en 1950, 40 en 1970, 37 en 2000 et moins de 35 heures en 2010. Cet auteur atteste également que $25 \%$ de la population est orientée à des valeurs éthiques du travail tandis qu'au moins les trois quarts adhèrent aux valeurs familiales, au loisir et à leur mieux-être ou à des rapports combinatoires de ces trois valeurs comme éléments centraux de leur vie quotidienne.

Volant (1976) affirme que la réalité sociale du loisir en évolution pose des questions sur le droit au travail et au temps libre. Avec l'apport de la technologie et de l'industrialisation, la lutte pour l'existence serait éliminée et les personnes seraient libérées du travail aliénant. D'après lui, la question à poser est de savoir qui a le droit de travailler et que fait-on de son temps libre? Ainsi, il existe un rapport de force entre le travail et le temps libéré du loisir qui sont des temporalités distinctes. D'une part, le travail est un temps obligatoire pour la survie personnelle et collective. Trop souvent, le travailleur est aliéné parce qu'il produit à l'extérieur de lui-même et ne peut prendre conscience véritablement de son action parce qu'il ne reconnait plus les motivations profondes et la finalité propre du sens interne de son action (Marx, 1996; Weil, 1949). Il s'aperçoit alors que celui-ci ne fait aucun sens si ce n'est qu'il répond à des raisons instrumentales. D'autre part, le loisir indique plutôt ce que l'on n'est pas obligé de faire sans chercher toujours à satisfaire démesurément les besoins primaires, parfois trop éphémères. De telle sorte que le loisir n'est qu'un moyen parmi tant d'autres à la réalisation personnelle. Il possède sa propre finalité interne cependant préférable à la vie active rémunérée parce qu'il autorise à réfléchir plus convenablement sur le sens de la vie lorsque le sujet détient les moyens financiers acceptables pour le faire. L'important est de rechercher les conditions nécessaires et les types d'occupations pour optimiser son temps à l'expérience du loisir. D'après les conditions sociales (sexe, âges, statut social, origine ethnique), le conflit sémantique et d'interprétation laisse entrevoir que l'expérience du loisir pour certaines personnes n'est pas obligatoirement de même intensité que pour d'autres personnes plus enclines à d'autres valeurs telles la famille, les ami(e)s, l'engagement politique et/ou spirituel/religieux. Un juste équilibre est requis. Émerge alors un certain nombre de questions : (1) Y aura-t-il pour les gens libérés du travail une façon de rester intégrés dans une vie de loisir?; (2) Le loisir ne cache-t-il pas le sentiment d'ennui et de solitude menant à des expériences aliénantes?; et (3) En d'autres termes, comment élaborer les voies d'une vie qui s'inscrivent dans le loisir porteur de sens véritable? 


\section{Tendance sociale \# 2 - Le vieillissement de la population}

Force est de constater que le vieillissement progressif de la population représente, pour aujourd'hui et demain, un défi de taille chez les personnes qui sont aux prises avec l'accroissement du temps libre. Pour nourrir la réflexion, deux générations font face à la problématique de l'accroissement du temps libre et du loisir. Ce sont les jeunes et les personnes aînées.

\section{Les jeunes}

Il existe une césure entre deux populations chez les jeunes. Il y a celle qui intègre le marché du travail avec les mécanismes corporatifs de défense au bien commun. Elle représente le progrès avec ses conceptions de vie positive que véhicule l'ensemble de la société. L'autre population est constituée de jeunes condamnés à la pauvreté, à l'aide sociale et au chômage sans profiter d'abri syndical. Rejetés par une société qui valorise la performance et la compétition à outrance, ils ne se conforment pas aux valeurs dominantes excessives que veulent imposer les plus performants de la société. Sans modèle de référence protecteur, ces jeunes rêvent de liberté, d'aventure et d'indépendance d'esprit. Ils sont à la quête de causes justes et d'espérance pour entrer dans la vie adulte. Les loisirs constituent une soupape ou à un temps d'évasion pour cette catégorie de population. Les jeunes de 15 à 24 ans profitent considérablement de temps libre qu'ils consacrent aux loisirs ainsi qu'à l'éducation et sont sous-représentés dans le monde du travail (Pronovost, 1997). Le loisir procure un contexte pour les jeunes pour développer leur identité personnelle, leur rôle social et leurs relations avec les pairs. Néanmoins, les jeunes peuvent être la cible pour les loisirs de [sur]consommation que l'on retrouve notamment dans le monde virtuel s'exprimant dans les vidéos de musique populaire, la musique et ses spectacles ainsi que des vêtements qui véhiculent des valeurs marginales. De même, les jeunes, plus particulièrement à l'adolescence, sont confrontés aux problèmes de consommation de tabac, de drogues et d'alcool, du stress, de l'ennui, de l'isolation, de l'embonpoint et des formes extrêmes de loisir qui peuvent contribuer à la violence telle que l'on observe sur Internet et dans les médias de masse.

Kraus (2000) affirme que le défi sera de promouvoir des valeurs positives et une pratique de la participation sportive par la jeunesse pour que tous soient gagnants. L'excellence dans une approche de compétition équilibrée contribue à former positivement le caractère des jeunes tel que l'autodiscipline, l'engagement à une cause, la loyauté, l'esprit sportif et le respect de l'adversaire afin que celui-ci nous respecte en retour. Surgissent ici à notre esprit quelques questions : (1) Quel sera le rôle des intervenant(e)s en loisir pour organiser des activités sportives et de loisir pour la jeunesse?; (2) Avec l'aide des parents et entraîneur(e)s, comment pourront-ils faire observer le respect aux règles et garder ainsi le sens de l'esprit sportif qui contribue à garantir une expérience positive chez les jeunes?; et (3) Comment lutter envers des pratiques de loisir aliénant sans adopter un ton moralisateur?

\section{Les personnes aînées}

Un fait probant est celui de l'émergence des personnes du troisième et du quatrième âge dans les divers réseaux de la société. La génération qui concerne les personnes aînées de 65 ans et plus montre une projection moyenne croissante pour les années à venir. Ainsi, le pourcentage des personnes aînées sera en 2021 de $18,9 \%$ et en 2031 de 23,6 \%. L'avancement de la retraite avant 65 ans, associé à la progression de l'espérance de vie 
à la naissance soit de 75,5 ans pour les hommes et de 81,2 ans pour les femmes libère le temps libre dans le parcours de vie de ce groupe d'âge spécifique (Statistique Canada, 2001). Les nouveaux retraités sont voués à un accroissement inévitable de temps libre. L'emploi à temps partiel est apprécié, mais ils subissent une exclusion au marché du travail (Guillemard, 2002). Brault (1990) atteste que la plupart des retraités sont des personnes autonomes, en bonne santé et presque les deux tiers d'entre elles ont des revenus acceptables, alors qu'en 1971, 57 \% des retraitées étaient sous le seuil de la pauvreté.

À noter cependant que les personnes de 65 ans et plus sont proportionnellement plus nombreuses à souffrir d'au moins deux maladies chroniques. Elles sont proportionnellement moins nombreuses dans la participation à des gestes de gratuité de type bénévole, mais lorsqu'elles y consacrent du temps, elles en font beaucoup plus que les autres dans cette catégorie d'âge (Institut de la statistique du Québec, 2012). Ceux qui ne participent pas à une activité bénévole préfèrent plutôt pratiquer le loisir touristique. Les retraités revendiquent la solidarité sociale et se situent dans « la polychronie et les investissements multiples dans le travail bénévole, les loisirs, la famille, la cité. [. . .] La retraite n'est plus cet âge de la vie-d'après-le-travail. Elle devient celle d'un âge où l'activité marchande fait place à l'activité libre. L'enchevêtrement des temps sociaux est devenu aussi la règle pour le troisième temps de la vie » (Guillemard, 2002, p. 65). Les personnes aînées portent en elles le devoir de retransmettre des valeurs souveraines et à affermir la mémoire collective d'une société donnée. Cela constitue une motivation importante à demeurer active dans la contemporanéité.

Deux catégories sociales de personnes aînées se distinguent (Kraus, 2000) : ceux qui sont en santé, optimistes, mobiles et indépendants et ceux qui ont des problèmes de santé ainsi que de mobilité affectant profondément leur qualité de vie. L'envers de la médaille au vieillissement est le suivant : dans une société orientée à surproduire, le risque que les personnes aînées soient considérées comme une valeur inutile et assujettie au processus de l'exclusion sociale constitue une possibilité dans l'idéologie utilitariste. Dans leur solitude, des personnes aînées face à eux-mêmes sont confrontées au sentiment d'ennui et d'isolement (Delisle, 1987). Elles peuvent expérimenter à leur corps défendant l'épiphénomène de l'âgisme parce que les membres productifs de la société les considèrent comme inutiles ou improductives, voire même nuisibles dans une société qui valorise démesurément la performance et la compétition. En même temps, des regroupements cherchent à déconstruire les représentations sociales négatives que la population active entretient des personnes aînées (Charpentier \& Quéniart, 2007). Le combat contre les préjugés personnels et sociaux est le quotidien de ces groupes de défense.

À travers le vieillissement, il y a un facteur inhérent qui détermine la qualité de vie. Ainsi, le mal du stress envahit la vie quotidienne des personnes vieillissantes ${ }^{7}$ et aînées étant devenu un symptôme médical à traiter. Pronovost et Henri (1996) affirment la perception du manque de temps chez les Canadien(nes). Les répondants de tous les âges ont le sentiment de ne pas avoir accompli ce qu'il voulait faire dans la journée; ils éprouvent du stress; ils se reconnaissent comme des bourreaux de travail; ils se sentent tendus parce qu'ils veulent plus d'activité; ils sont enfermés dans une routine quotidienne; ils s'inquiètent qu'ils ne consacrent pas assez de temps à leurs familles ou à leurs amis; ils manquent de temps pour s'amuser et ils diminuent même leurs heures de sommeil. Ces paramètres correspondent aux études américaines de Robinson et Godbey (2000) qui concluent que les personnes vieillissantes et aînées ont la perception, encore plus aujourd'hui qu'il y a 30 ans, de se sentir toujours pressés et qu'ils n'ont pas assez de temps de faire toutes les activités qu'ils veulent ou devraient faire. Ce mal du stress les soumet à une vie accélérée ne respectant pas le rythme naturel du métabolisme humain. Dès lors, il est 
souvent la cause de maladie et de détérioration de la santé mentale ${ }^{8}$ (Godbey, 1997). Les coûts pour maintenir le système de la santé en capital humain et financier sont maintenant onéreux dans les sociétés existantes. Quelques questions émergent à notre entendement: (1) Où faut-il agir pour réduire efficacement le mal du stress chez les personnes vieillissantes et aînées?; (2) Quelles seraient les priorités pour une politique sociale face à cette problématique d'envergure?; et (3) Faudrait-il adopter des mesures plus dissuasives vis-à-vis des comportements destructeurs non souhaitables qui engendrent le mal du stress?

\section{Tendance sociale \# 3 - Les valeurs qui font vivre}

Sans adopter une approche conventionnelle et arbitraire, nous identifions trois valeurs auxquelles les personnes sont prêtes à défendre et à lutter farouchement pour l'équilibre des valeurs humaines fondamentales. Ce sont les valeurs familiales, les valeurs de [sur]consommation et les valeurs du tiers secteur. Ces valeurs ont foncièrement des implications sur le loisir (Godbey, 1997).

\section{Les valeurs familiales}

La famille forme le contexte social commun du loisir. Les attentes de rôle et les ressources d'allocations du ménage reflètent les changements d'objectifs à travers le parcours de vie aussi bien que les effets psychosociaux de la structure familiale (Kelly, 1987). Or, la famille n'est plus ce qu'elle était confrontée aux taux relatifs de divorce dans un rapport de 1 sur 2 depuis la révolution sexuelle des années 60. Il existe maintenant des familles alternatives (unions libres, monoparentales, recomposées, familles d'accueil et familles adoptives) ainsi que des adultes expérimentant le sentiment de solitude (Kelly \& Freysinger, 2000). Les rapports amoureux entre les sexes changent depuis que la gent féminine a intégré le marché du travail et qu'elle parvient à assurer son indépendance économique bien que la lutte pour l'équité salariale ne soit pas atteinte présentement. Quelques questions demeurent ouvertes : (1) Est-ce que le taux de divorce augmentera avec la remise en cause constante des valeurs humaines, familiales et/ou spirituelles/religieuses?; (2) Y aura-t-il un retour à des formes plus traditionnelles de vie à deux?; et (3) Est-ce que le désir d'avoir des enfants continuera à se maintenir?

Même s'il existe différentes typologies familiales, les rôles avec ses responsabilités et ses prises de décision ont des conséquences sur le loisir. Dans les contextes situationnels, les divertissements à la maison, la planification des vacances, le soutien aux activités des enfants, les contraintes de l'horaire scolaire, la participation aux organisations bénévoles, la gestion de l'espace du foyer et pour toutes les choses qui vont de la télévision à l'intimité sont des activités qui interagissent sur le loisir (Kelly, 1987). Du reste, l'expression proverbiale : Une famille qui joue ensemble restera ensemble, et en bonne santé est un modus vivendi de la vie à deux et favorise le développement de l'intimité, de l'amitié et de l'épanouissement à part entière des membres de la famille. Une donnée factuelle demeure: la diversité des formes de famille change le visage du loisir. Le travail a également un impact sur la famille et le loisir. La réduction des heures hebdomadaires de travail dégage du temps au loisir familial. Cependant, la distance grandissante entre les lieux de travail et la résidence occasionne des coûts en termes de temps et des restrictions à ce type de loisir. Cet état de choses suggère quelques questions supplémentaires : (1) Le temps alloué au travail déterminera-t-il la dynamique propre de la vie en famille?; (2) Comment développer des conditions d'emploi justifiant d'optimiser le loisir familial?; et (3) Comment faire pour que la famille devienne une valeur partagée dans la société canadienne? 
Les membres de la famille allouent passablement de leur temps libre qui va de l'écoute de la télévision jusqu'à l'utilisation substantielle de l'Internet (Robinson \& Godbey, 2000). Cette utilisation apporte un divertissement accessible peu coûteux pour la famille et constitue un chemin important par lequel les informations lui parviennent. D'autant plus que le monde du spectacle, du sport et du tourisme fait partie intégrante de la commercialisation par la télévision, de l'Internet ou les appareils cellulaires qui donnent des possibilités d'accéder aux autres personnes d'une manière instantanée. Il est à se demander si ces outils technologiques n'entraînent pas les personnes dans un monde factice des apparences et de la séduction en suscitant un hyperindividualisme exacerbé qui les attire sournoisement dans un monde virtuel hors du temps réel (Lachance, 2011; Levy, 1995; Lipovetski, 1983, 1987; Schleifer et coll., 2010). À cela, la télévision entre autres choses contribuerait largement à cette tendance fantasmagorique. Elle recoupe un monde symbolique propre à chaque génération et créé des liens significatifs entre elles. Pendant un instant, l'impression aux téléspectateurs de dominer le monde sans rencontrer aucun obstacle entretient leur réalité existentielle (Ricard, 1992; Wunenburger, 2003). La même dynamique est constatée chez ceux qui passent de nombreuses heures à naviguer sur le réseau Internet. Projeté sans en prendre réellement conscience dans un monde virtuel et imaginaire, l'utilisateur est alors coupé de la réalité du monde. Isolé des autres, il risque de perdre tout contact avec le réel. Quelques questions ont préséance : (1) La télévision ne devient-elle pas le miroir magique qui, au lieu d'informer, séduit la naïveté du téléspectateur et le berce d'illusion en le renvoyant à sa suffisance égotiste et alimente en informations de type fétichiste les personnalités narcissiques?; (2) Comment diminuer les impacts funestes qui déstructurent le noyau familial des membres cherchant à demeurer ensemble en toute harmonie?; et (3) Comment lutter envers les valeurs hyperindividualistes qui modifient la personnalité humaine?

\section{Les valeurs de [sur]consommation}

Le loisir de [sur]consommation possède une valeur symbolique qui sert à distinguer les classes sociales supérieures de la culture de masse. Il prend alors une valeur ostentatoire (Veblen, 1899). Dans cette rationalité, le loisir de [sur]consommation est au service de finalités instrumentales de possession. La personne cesse d'être considérée comme une personne. Elle devient plutôt l'image inerte d'une classe sociale qui la dépossède de son âme par une consommation extrême des loisirs. Lipovetski (1987) affirme que le loisir de [sur]consommation détermine la programmation au quotidien, elle manipule la vie individuelle et sociale, tout risque de verser dans un monde superficiel et évasif. En outre, ce loisir de type marchandise encourage la [sur]consommation de masse et improductive de temps en promettant un bonheur fragile et illusoire.

En dépit des soubresauts économiques du système capitaliste, les dépenses des ménages en matière de loisir sont non-négligeables soit près de $20 \%$ du revenu par ménage (Pronovost, 1997). Ceux-ci favorisent sa propre démocratisation, et en même temps, la culture de masse. Toutefois, il est difficile d'éluder les effets pernicieux de la société de [sur]consommation et du phénomène de l'aliénation des loisirs qui se déterminent par la logique de l'éthique du profit. D'ailleurs, tout système qui définit les personnes en termes de possession plutôt que d'action susceptible de réaliser leur vie est totalement aliénant, dans les loisirs aussi bien que dans le travail (Kelly, 1987, 2012; Marx, 1996). La violence symbolique surgit pendant le temps des loisirs, lors des fêtes ou à l'occasion d'activités sportives. L'agressivité verbale, physique et psychologique des entraîneurs, des parents, des partisans et des athlètes eux-mêmes lors des événements sportifs sont inquiétantes 
par leur omniprésence. Dans la tourmente et la frénésie de la compétition, les personnes entretiennent la rage de gagner et l'esprit de compétition destructeur, où l'autre n'a pas sa raison d'être ni sa place pour vivre. Les sportifs cherchent ainsi à s'affirmer pleinement par la domination outrancière de leurs adversaires. Il s'agit d' 'éliminer' les autres, de les 'battre' et de les 'écraser' à coup sûr (Bouet, 1969). À cet effet, le leitmotiv est, la fin justifie les moyens!

Par ailleurs, le terrorisme international est maintenant une dure réalité et frappe la conscience collective au quotidien en devenant une nouvelle forme de guerre (Godbey, 1997). Les personnes sont intimidées par une peur transmise du fond des âges. Il est en partie une réaction d'une culture vis-à-vis d'une autre culture qui s'oppose à un loisir aliénant de [sur]consommation de type fétichiste (Kelly, 1987). La sécurité nationale fait partie maintenant de l'ordre du jour et, en même temps, on assiste à une baisse significative de l'achalandage du tourisme international. Il devient important d'approfondir la question du loisir dans sa contribution à l'intégration entre les cultures. La pratique du loisir et des sports sont toujours des moyens efficaces pour créer la paix et éviter la dérive des tensions conflictuelles. L'exemple des Jeux olympiques qui se sont déroulés sans incident catastrophique depuis le massacre de Tlatelolco commis quelques jours avant la cérémonie d'ouverture aux Jeux de Mexico (1968) et de la prise d'otages aux Jeux de Munich (1972) est une bonne illustration, où les événements de loisir international de type sportif deviennent des lieux de pacification entre les cultures. Dans un tel contexte, des questions se posent pour les intervenant(e)s en loisir : (1) Quelle sera la nouvelle finalité du loisir face à la [sur]consommation?; (2) Quelle sera la place du loisir dans l'intervention municipale et les institutions politiques?; et (3) Quels seront les dispositifs concrets et pacifiques mis en place pour diminuer la peur dans l'environnement social?

\section{Les valeurs du tiers secteur}

La révolution de l'informatique, de la bureautique et des télécommunications grâce au progrès technoscientifique a rendu les machines plus performantes. Cet état de fait comporte une influence déterminante sur le temps libre et le loisir. Le temps libre gagne du terrain particulièrement en raison du taux de chômage élevé. Rifkin (1995) prétend que l'on assiste sans pouvoir réellement agir au déclin du monde de l'emploi des sociétés postindustrielles. Il émet l'hypothèse du chômage technologique qu'il nomme 'la troisième révolution industrielle'. Des millions de personnes sont vouées aux chômages et à la misère humaine pendant que les états ne savent pas comment arrêter cette mondialisation technologique qui bouleverse les entreprises et remplace les travailleurs par des machines. Les personnes doivent se requalifier pour s'adapter à la nouvelle technologie pendant que les industries-employeurs ne peuvent répondre à la férocité de la compétition internationale et n'ont d'autre choix que de fermer leurs portes. Le constat de Rifkin (1995) est pessimiste parce que la montée du chômage mondial creuse l'écart entre les riches et les pauvres et provoque ainsi un climat d'incertitude, voire même d'une lutte des classes sans précédent entre les pays. Cet auteur croit que la concurrence mondiale et la rationalisation des secteurs marchands publics et privés vont affecter la vie des travailleurs selon deux directions. Ceux qui garderont un emploi devront faire face à la diminution du temps de travail et profiteront alors d'un accroissement de temps libre et ceux qui sont chômeurs subissant un temps inoccupé. Pour sa part, Kelly (2012) avance que la production dans sa perspective globale signifie qu'il y aura moins de sécurité d'emploi pour les travailleurs puisqu'il n'y a aucune confirmation que les professions, les emplois et les carrières qui existent déjà demeureront dans la présente forme dans 10 ou 20 ans. 
Schnapper (1997) réfute au contraire l'éventualité de 'la fin du travail' puisqu'un nouvel équilibre mondial s'établit par la création d'autres emplois due à la révolution informatique, de la diminution du temps de travail et des tâches moins pénibles que subissent les travailleurs en raison des nouvelles machines. Le monde du travail est plutôt en période d'ajustement pour une meilleure pérennité. Au-delà des présupposés théoriques de 'la fin du travail' ou de 'la nouvelle organisation du travail', il faudrait admettre que cette réalité du chômage technologique est un processus qu'on observe depuis le commencement de l'ère capitalisme. L'économie de marché demeure ouverte et concurrentielle. Les emplois ne cessent de disparaître et d'autres restent à imaginer. En ce sens, Toffler (1980) affirme que beaucoup de changements sociaux se produisent indéfiniment en peu de temps, où la société de la troisième vague dite post-industrielle (après 1950) se caractérise par le flux et l'influx rétroactif de l'information, de la technologie et de la diversité grandissante des sous-cultures. Ces états de fait ont un impact sur le travail puisque plusieurs personnes centralisent cette activité humaine à partir du bureau de leur résidence privée et que la télématique est l'outil de travail de prédilection.

Rifkin ainsi que Schnapper mentionnent également l'importance de réfléchir sur les formes de liens sociaux qui ne sont pas seulement ceux suscitant la participation à la production concurrentielle. En ce sens, ils signalent toute l'importance de reconnaître l'utilité de l'activité gratuite qui serait un moyen efficace pour déployer les compétences non exploitées de millions de personnes dans des fonctions valorisantes. Cette 'troisième force' se traduit nécessairement par l'action bénévole que l'on nomme 'le tiers secteur' ou l'économie sociale.

Hall et al. (1998, p. 11) affirment que le nombre annuel moyen d'heures données par les bénévoles a décliné au cours des 10 dernières années. Le bénévole a offert en moyenne 149 heures en 1997, comparativement à 191 heures en 1987 (Hall et al., 1998, p. 11). $\mathrm{Au}$ reste, près des trois quarts des bénévoles ont exprimé que le manque de temps a un impact négatif sur leur temps accordé à l'action bénévole. De son côté, les organisations de loisir accueillent près de 500000 bénévoles (Thibault \& Fortier, 2003). Ceux-ci dépendent de l'engagement d'intervenant(e)s assumant le fonctionnement et le développement au loisir particulièrement en milieu scolaire et municipal. Ajoutons à cette réalité de l'action bénévole, le manque d'appui financier et les exigences plus élevées de la population devenue 'cliente' au fil des changements politiques, sociaux, culturels, religieux et générationnels (Thibault, Albertus, \& Fortier, 2007). Le bénévolat de type 'clientéliste' éloigne trop souvent les personnes qui désireraient donner de leur temps libre.

Par contre, d'autres données quantitatives montrent au contraire que ce type d'activité se porte bien. Les États-Unis, par exemple, est considéré le premier pays du monde, où 93 millions d'Américains le pratiquent à divers degrés (Bénévolat, Wikipédia, http://fr. wikipedia.org/wiki/Bénévolat). Il implique surtout les retraités et les femmes. Vézina et Crompton (2012, p. 39) révèlent qu'en 2010, plus de 13,3 millions de personnes, soit $47 \%$ des Canadiens (15 ans et plus) ont participé à l'action bénévole soit près de 2,07 milliards d'heures dont le volume de travail équivalent à un peu moins de 1,1 million de postes à temps plein. Cette enquête pancanadienne démontre que la plupart des bénévoles $(93 \%)$ sont motivés afin de contribuer à leur communauté associative immédiate. Près de la moitié des bénévoles (43\%) ont déclaré qu'ils ont donné du temps dans le cadre d'un projet de groupe avec des amis, des voisins ou des collègues. La grande partie des heures totales à l'action bénévole (66\%) sont consacrées à cinq types d'organismes de bienfaisance à but non lucratif : les sports et les loisirs (19\% des heures aux totales), les services sociaux (18\%), la religion (15\%), l'éducation et la recherche $(9 \%)$ et à la santé $(5 \%)$. Fait à noter, ce sont les jeunes qui sont plus enclins à pratiquer l'action bénévole; 
et que l'on retrouve souvent les mêmes personnes qui se dévouent pour leur communauté sociale. Pour résumer cette section des valeurs qui font vivre, certaines questions liées au loisir nécessitent un temps d'arrêt : (1) Le loisir de [sur]consommation sera-t-il seulement une affaire de dépense ostentatoire définissant les paramètres des classes sociales?; (2) Est-ce que la [sur]consommation et les effets du chômage technologique transformeront la manière de pratiquer le loisir?; et (3) L'action bénévole pourra-t-elle devenir un modus vivendi pour ceux qui profitent suffisamment du temps libéré du loisir pour le pratiquer?

Les tendances sociales de l'accroissement du temps libre, du vieillissement de la population et des valeurs qui font vivre requièrent une réflexion plus pointue dont découlent trois enjeux du loisir pour la régénérescence du tissu social. Cette prise de conscience plus aiguisée est de la plus haute importance pour le développement des cultures de la contemporanéité devenue non monolithique. Il s'agit de faire disparaître certaines contrariétés qui freinent l'épanouissement des personnes dans la perspective d'une meilleure santé mentale à visée optimale.

\section{Les enjeux du loisir}

Les tendances sociales identifiées soulèvent un questionnement prospectif autour de trois enjeux du loisir décisifs pour notre temps et sollicitent de co-élaborer le sens pour la régénérescence du tissu social. L'action bénévole, le loisir thérapeutique et l'éducation au loisir sont les points d'ancrage à l'échafaudage du loisir afin que les citoyen(ne)s en sortent gagnant(e)s dans leur vie quotidienne.

\section{Enjeu du loisir \# 1 - L'action bénévole}

Nous commençons à peine à considérer l'action bénévole comme un phénomène social important à maintenir pour des raisons d'humanisation des personnes. Tocqueville suggère ceci : " pour que les hommes restent civilisés ou le deviennent, il faut que parmi eux l'art de s'associer se développe et se perfectionne dans le même rapport que l'égalité des conditions s'accroît » (de Tocqueville, 1968, p. 258). L'action bénévole est un geste pratique qui prend sa place dans les communautés associatives. Les personnes engagées dans ce mouvement ont l'impression de participer dans une dynamique éminemment politique et ne considèrent pas leur action comme étant un geste oisif et inutile, mais plutôt comme une forme de militantisme dans la vision d'une société alternative (Baum, 1999). Par exemple au Québec, Dumont (1995) certifie ce mouvement parallèle de société qu'est l'action bénévole qui a représenté un facteur déterminant de changement social sans précédent depuis la Révolution tranquille puisque cette société s'est reconstruite par le bas après avoir été ébranlée dans ses principes chrétiens et laïcs.

D'après Weil (1949), chaque personne aspire d'avoir plusieurs racines en reconnaissant la presque totalité de sa vie morale, intellectuelle et spirituelle par l'entremise de la communauté sociale à laquelle elle participe à partir de son appartenance naturelle. Ainsi, les personnes seront de plus en plus interpelées à s'engager à l'action bénévole, sinon à des gestes de gratuité ponctuels. Chacun aura à choisir ce type d'activité qui tienne compte de ses besoins physiques et moraux, de ses compétences et de sa disponibilité dans une société en changement. L'action bénévole sera un lieu privilégié, où les membres d'une société se responsabiliseront d'eux-mêmes malgré ses structures institutionnelles ou organisationnelles parfois inaccessibles, impersonnelles, voire même déshumanisantes. 
D'après Masson (1991), le monde des 'petites patries' renaît et l'expression populaire Small is Beautiful (Schumacher, 1978) redevient l'expression à la mode avec cette observation de Tocqueville (1968, pp. 253-254) : «Les Américains de tous les âges, de toutes les conditions, de tous les esprits, s'unissent sans cesse. [. . .] S'agit-il enfin de mettre en lumière une vérité ou de développer un sentiment par l'appui d'un grand exemple, ils s'associent ». À partir de là, les citoyen(ne)s des divers lieux de société seront en mesure de maîtriser adéquatement leur environnement social. Ils seront prêts à s'engager, à donner de leur temps au bien commun par un engagement qui prend des formes d'action volontaire selon les types de personnalité spécifiques.

L'action bénévole pose des questions de santé mentale et concerne nécessairement les liens qui se tissent entre les générations. Dumazedier (1990b) avance que la nouvelle situation historique du vieillissement de la population concerne désormais tous les membres de la société dans la recherche de nouvelles stabilités entre les temps sociaux et les rapports intergénérationnels. Les personnes aînées sont créatrices d'une société en mouvement, d'une culture et d'une économie renouvelée pour autant que les liens conviviaux et solidaires se tissent entre les diverses strates générationnelles. L'action bénévole se caractérise par une recherche de réalisation, de pouvoir et d'affiliation (Henderson, 1981). Elle prend diverses formes d'activités volontaires dans les communautés associatives et locales. ${ }^{10}$ Autrement dit, elle est une solution tout espérée pour réaliser l'intégration sociale inclusive. Ainsi, des rencontres se réalisent afin de transmettre, de créer des liens durables et d'établir une synergie à connotation spirituelle par l'entremise du dialogue citoyen. Celui-ci privilégie l'engagement social contribuant au développement des valeurs civiques et démocratiques, au renforcement et à la valorisation des institutions avec les pouvoirs politiques en présence ainsi que du rôle socio-économique prépondérant des mouvements coopératifs qui construisent les bases de la société humaine (Béland, Bouchard, \& Girard, 2012; Fahmy et coll., 2012). Cela dit, les interrogations sur les modes de relation sentimentale et émotionnelle, d'échange économique ainsi que de solidarité sociale se posent nécessairement pour que les citoyen(ne)s se sentent interpelé(e)s à s'engager à travers leur bien-vieillir faisant en sorte qu'ils(elles) soient toujours en quête d'un mieux-être porteur de sens.

Dans cette visée éthico-spirituelle, il est indéniable que les retraité(e)s possèdent des qualités exceptionnelles, bon nombre ont plusieurs années d'expérience et tous, plus de temps libre que n'importe qui. Pour certains, la participation citoyenne de cette strate générationnelle est une solution tout indiquée qui établirait de nouveaux rapports au temps en vue d'un effet collatéral bénéfique contribuant fortement à la régénérescence du tissu social. Chacun pourra choisir un engagement bénévole qui tient compte de ses besoins physiques et moraux, de ses compétences et de sa disponibilité dans un contexte social qui s'apprête à les accueillir. La construction de cette société requiert donc le renforcement du mouvement associatif et témoigne de la certitude que le geste de donner librement constitue la finalité ultime au bien commun.

Face à l'accroissement du temps libre, du vieillissement de la population et des valeurs qui font vivre, il s'agit de prendre conscience des priorités de l'existence afin de rendre le monde meilleur consistant à s'occuper des pauvres, c'est-à-dire en fournissant l'habitation, la nourriture, les soins élémentaires, l'éducation et la protection de l'environnement. Il s'agit de transformer pour le mieux la manière dont les personnes participent à la vie sociale. En ce sens, Rifkin (1995) propose des recommandations pour inspirer l'action bénévole. L'État pourrait développer un programme de déduction d'impôt pour les heures dispensées par l'acte bénévole, attribuer un salaire incitatif au bénévole notamment aux chômeurs à long terme (au lieu des primes de l'assurance emploi), développer des 
programmes de subvention aux organismes du tiers secteur pour leur donner les ressources humaines et matérielles nécessaires à actualiser le geste bénévole, encourager la réduction du temps de travail hebdomadaire, taxer le monde de la [sur]consommation du loisir notamment l'industrie informatique et électronique, les sport-spectacles et les spectacles d'humour pour redistribuer les argents au tiers secteur, et finalement, accorder un revenu annuel garanti (salaires virtuels) aux moins nantis qui s'efforcent à mettre en pratique l'action bénévole.

Les bénévoles sont des agents multiplicateurs ayant une fonction déterminante à exercer dans la société. L'ajustement d'une politique de l'action bénévole nous semble incontournable parce que la création de la communauté sociale est forcément une entreprise collective. Celle-ci est appelée à jouer un rôle important dans la quête d'identité des personnes. Elles y développent leur identité pouvant se définir comme un ensemble de relations fondées sur les interactions symboliques, la communication et le partage des tâches entre tous et chacun. L'action bénévole comme loisir est un lieu dynamique d'ouverture au monde entre les personnes tout en respectant l'individualité profonde qu'entretiennent les personnes dans leurs interactions. C'est ainsi qu'un loisir de type bénévole est le lieu privilégié dans le dessein d'aider le processus d'humanisation.

Dans l'espace social du loisir, les personnes se comportent dans le monde et apprennent qui elles sont dans leur vie quotidienne. À travers l'action bénévole, le mot d'ordre n'est pas 'avoir plus', mais bien 'être plus'. Dès lors, cette pratique est perçue non pas comme un simple moyen, mais détient sa propre finalité au cœur de l'aventure humaine parce qu'elle est associée aux notions d'authenticité et de liberté assurant à celui qui le pratique des sentiments de repos et d'émotions de joie. Elle est à la fois gratuité, compassion, altruisme et générosité. C'est que l'action bénévole, au-delà de l'utilitarisme rationnel, est un don. Comme instrument de changement dans la société contemporaine, elle participe implicitement au ré-enchantement et à l'indétermination du monde (Caillé, 2007; Godbout 2007; Godbout \& Caillé, 1992; Robichaud, 1998) de sorte que l'action bénévole est par essence le geste par excellence qui fait exister et participer implicitement les personnes à la création de leur communauté sociale. On donne pour le plaisir même de donner, pour le plaisir que cela génère à l'autre, pour l'amour de l'autre et pour le partage des mêmes intérêts pour le plus grand nombre. Dans cet horizon de pensée, « notre identité se construit dans la mesure où nous rendons actif ce que nous avons reçu en donnant à notre tour » (Godbout, 2007, p. 367). Il existe donc une joie d'existence à donner de soi pour les autres afin de se libérer des souffrances et de rendre ainsi notre environnement plus viable et harmonieux.

Quoi qu'il en soit, l'action bénévole n'est pas totalement gratuite. Le bénévole cherche consciemment et/ou inconsciemment une contrepartie à son don c'est-à-dire un contredon. De fait, les personnes recherchent quelques bénéfices en retour du geste gratuit posé même si cela n'est pas réellement voulu au départ. Les rapports entre donner, recevoir et rendre deviennent alors les éléments essentiels à l'action bénévole créant son propre mouvement. L'acte symbolique interactif du don créé le lien social, où les bénévoles « sont des signes de signes, des relations de relations entre signes » (Caillé, 2007, p. 209). Il existe donc une panoplie de mondes symboliques par le simple geste gratuit qui s'inscrit dans le cadre formel de « la triple obligation de donner, recevoir et rendre qu'une chose peut valoir pour une autre. C'est parce qu'une chose est donnée, ou susceptible d'être donnée, qu'elle fait sens. Il n'est de sens que du don » (Caillé, 2007, p. 216). Il s'agit alors de trouver des lieux, où il est permis de faire circuler librement ces notions pour que tous y retrouvent son compte afin d'exister pleinement comme personne. Ils se créent alors de nouveaux liens et espaces sociaux à travers l'idéal communautaire s'enracinant dans la culture des sentiments et de la complétude des émotions. 
Les municipalités de même que les groupements associatifs qui accueillent des bénévoles devront s'ajuster aux diverses valeurs et motivations guidant les actions citoyennes. On s'engage autant pour la cause sociale et l'entraide que pour le plaisir ou l'apprentissage que nous rapporte cet investissement de son temps donné. Une adaptation de la gouvernance des organisations en fonction des styles de vie qui caractérisent nos sociétés actuelles, associées à une concertation et un réseautage entre acteur(actrice)s, deviendront de premières nécessités à la qualité, voire à la survie de cette forme de loisir qu'est l'action bénévole. Il peut même constituer un moment important dans le cadre du travail rémunéré (Haworth \& Lewis, 2005) dans des conditions bien précises comme le temps supplémentaire ou le service à la communauté sociale. Il s'agit de poser de multiples actions concrètes pour recruter les bénévoles et préparer la relève. Ainsi, l'action bénévole devra tendre vers une vocation collective que l'État a la responsabilité morale d'organiser. À cet égard, la connaissance notionnelle des valeurs et des motivations chez les bénévoles à savoir pourquoi ceux-ci posent des gestes gratuits se révèle fondamentale dans la démarche compréhensive du cadre théorico-pratique au loisir (Lockstone-Binney, Holmes, Smith, \& Baum, 2010). Les intervenant(e)s en loisir quant à eux auront à développer des stratégies et des activités d'apprentissage chez leurs bénévoles (Harvey, 2003) parce que le 'clientélisme' influence la dynamique de l'action bénévole et l'initiative citoyenne comme expérience de loisir (Thibault et al., 2007). Les bénévoles effectuent un nombre croissant de tâches, de plus en plus lourdes, pour offrir à la population québécoise des services publics diversifiés et de qualité. Cependant, ils s'essoufflent et le recrutement devient ardu et difficile. Un autre défi de cette forme de lien social consistera à l'amélioration des compétences et des aptitudes du personnel rémunéré en gestion de bénévoles (Gagnon \& Sévigny, 2000). Une politique de l'action bénévole en devenir nous semble être une voie incontournable. Cela soulève quelques questions de pointe : (1) Quelles seront les actions à poser à court et moyen terme afin de gérer efficacement le geste de gratuité des bénévoles?; (2) Quelles seront les mesures pour assurer le recrutement, la formation et la reconnaissance des bénévoles?; et (3) Quels seront les rôles des politiciens et des intervenant(e)s en loisir en matière d'action bénévole?

\section{Enjeu du loisir \# 2 - Le loisir thérapeutique}

Le loisir thérapeutique demeure un facteur important pour la prévention dans la quête d'une santé équilibrée plus effective. Lord et Hutchison $(1979,2007)$ mentionnent toute l'importance du loisir en termes de coûts-bénéfices auprès des personnes vulnérables en établissant les conditions nécessaires socialement favorables et acceptables pour que celles-ci soient des membres à part entière dans la communauté. Cela dit, elles seront plus en mesure de contribuer à la vie sociale pour un monde meilleur et plus en santé. Au cours de ces dernières années, il y a eu émergence de projets sociaux innovateurs qui reflètent une nouvelle manière de penser, où tous les acteur(actrice)s apprennent à créer leur voie en affirmant : « Nous créons notre chemin en y marchant 》 (Lord \& Hutchison, 2007, p. 8, trad.). La communauté et les interactions par le biais d'intérêts partagés autorisent d'ouvrir la voie vers un processus social inclusif qui autorise la facilitation au loisir chez les personnes vulnérables. Il ne s'agit pas de mettre en place tout un système « de livraison à domicile ou de développer toujours des programmes pour eux. C'est surtout de fournir des opportunités afin de développer leur propre vie et leur temps de loisir. [ . . ] Cela signifie de 'permettre' plutôt que de 'délivrer' étant le concept fondamental. La facilitation au loisir commence avec l'acceptation sociale et l'adaptation des opportunités » (Kelly, 2012, p. 491, trad.). Cela requiert aussi des programmes ajustés mettant l'emphase au 
modèle de counseling personnalisé. Dans cette foulée, Loesch et Wheeler (1982) développent un cadre théorique de counseling adaptable au loisir thérapeutique. Le réseautage, l'exploration, l'action et la finalisation sont les étapes qui améliorent le processus d'un 'état d'esprit' positif dans la perspective individuelle, groupale et développementale. Les habiletés telles que l'empathie, le regard optimiste, la congruence et le respect sont mises de l'avant établissant l'équilibre des dimensions affectives (c.-à-d. les sentiments/les attitudes/les valeurs/l'attente/les intérêts), et ce, pour l'évaluation de l'expérience en loisir.

Searle et Brayley (2000) apportent un discours sur la réalité des personnes vulnérables de la société. Ils abordent des problèmes et des défis que sont confrontés les intervenant(e)s en loisir dans une société complexe et vieillissante qui recherche une typologie de loisir qui ne soit pas sa propre finalité, mais considéré plutôt comme un moyen pour une plus grande fin. Toutefois, le loisir est menacé par l'absence de politique nationale au Canada. Karlis (2004) réaffirme que les pratiquants du loisir, les administrateurs, les professionnelles, les académiciens et les étudiant(e)s sont des agents multiplicateurs apportant des lumières sur la conscience humaine en termes d'opportunité, d'accessibilité, de reconnaissance sociale dans un pays qui prétend résoudre des questions ontologiques sur la liberté humaine dont le loisir est déjà un porte-étendard.

Lamari et Ménard (2012) décrivent les enjeux de l'économie sociale auprès des populations spéciales (c.-à-d. les familles à faible revenu, les personnes avec handicapes physique et mentale, les itinérants et les démunis, les personnes aînées). Ces catégories sociales ont besoin de circuler librement dans les facilités de sport et de loisir. À cette fin, l'assentiment de l'opinion publique est primordial bien que cela engendre « des pressions visant à assurer l'accès à toutes les personnes défavorisées, entraînant une hausse potentiellement importante des coûts, alors que l'enveloppe budgétaire est actuellement limitée et qu'aucune augmentation n'est prévue » (Lamari \& Ménard, 2012, p. 51). Pourtant, l'optique à transmettre de l'espoir au sans espoir est une condition importante à la régénérescence du tissu social.

Le loisir thérapeutique est un facteur primordial de résistance combattant les stéréotypes de la démence en ralentissant le processus mortifère du vieillissement. Il offre également des voies salutaires à une expérience synergique de type psychothérapeutique. Par les modalités de résistance affirmées, les personnes peuvent chercher à renforcer leurs forces intérieures et changer ainsi les discours négatifs à l'égard des idéologies dominantes (Genoe, 2010). L'important sera de résister avec les moyens disponibles dans la légalité et le respect des parties concernées pour que tous soient gagnant-gagnant.

Les milieux de santé sont déjà convoqués au loisir thérapeutique (Deschênes, 2002; Heintzman, 2008; Roussel, Ouellette, Singleton, \& Hall, 2012), où agissent déjà des techniciens d'intervention en loisir, des récréologues, des animateurs thérapeutiques, des techniciens d'éducation spécialisée avec des tâches professionnelles spécifiques s'exécutant dans les centres d'hébergement, les résidences privées, les centres de réadaptation, les centres psychiatriques et les centres de jour. Ces thérapeutes du loisir auront à construire la part qu'ils leur reviennent concernant la gérance des bénévoles engagés dans ces milieux institutionnels. Pour le reste, l'augmentation croissante des personnes aînées en perte d'autonomie (c.-à-d. affection psychique/démence/errance/déficits cognitifs ou physiques/perte affective) requiert des soins urgents et adaptés. Bédard et Carbonneau (2012, p. 48) « confirment les besoins de formation en loisir des propriétaires et des intervenants de résidences privées pour les personnes aînées ». Les problématiques du vieillissement de la population en termes de qualité de la vie seraient ainsi partiellement résolues par l'entremise des propriétaires et des 
intervenants qui offrent une programmation d'activités physiques et de loisir en résidences privées.

Cet enjeu du loisir laisse aux personnes vieillissantes et aînées d'être actives physiquement et productives socialement. L'exercice physique, une bonne nutrition, un contrôle des médicaments, du tabac, des drogues et de l'alcool sont les premiers pas vers l'atteinte d'un état de mieux-être par la mise en pratique du loisir thérapeutique. Ce mouvement en gestation applique cet adage philosophique Un esprit sain dans un corps sain. Or, si l'absence de maladie est signe de santé, alors les personnes aînées qui sont activement impliquées dans la vie sembleront plus jeunes d'esprit autant que les personnes vieillissantes participant à la vie productive. Autrement dit, les personnes vieillissantes et aînées qui sont productives demeurent en santé, et si elles demeurent en santé, elles seront ainsi mieux en mesure d'être productives. Que ce soit un travail rémunéré ou non rémunéré, ils devraient exister des mesures compensatoires à seule fin d'envisager des opportunités pour garder les personnes vieillissantes et aînées actives tout en s'écartant de ce qui est malsain. Les conditions nécessaires ne manquent pas. Cela part de la bonne volonté de tous et chacun afin de transmettre ce sentiment de mieux-être en établissant des jalons préliminaires en prévision de demeurer en bonne santé mentale en vue d'une vie réussie sans mesures hétéronomiques excessives.

Le loisir thérapeutique est un acte avec son histoire, son interprétation, son développement et sa visée éthico-spirituel. Il existe des défis à surmonter le mal ambiant en vue de construire l'univers de sens du loisir dans lequel nous voulons vivre parce que «l'univers de la personne, c'est l'univers de l'homme » (Mounier, 2010, p. 7). En ce sens, les personnes vieillissantes et aînées seront appelées à une transformation personnelle qui sollicite à l'engagement et à la responsabilité, où la jonction avec les événements révéleront et concrétiseront leur double et solidaire vocation intériorisée, c'est-à-dire apprendre à se recentrer afin de mieux s'épanouir (Mounier, 2010; Salomé, 1999). Par là, les personnes vieillissantes et aînées auront la possibilité de participer à la vie sociale de l'avenir permettant la circulation de valeurs sûres et durables.

Le loisir thérapeutique constitue un moyen efficace afin de prévenir l'effet maximal des problèmes notoires de santé, car sa visée éthico-spirituelle cherche à établir la santé par la motivation récréative pour une meilleure qualité de vie et l'atteinte d'un sentiment de mieux-être (Deschênes, 2011; Grafanaki et al., 2005; Heintzmann, 2008; Martin, 1975). À cette finalité, il est permis de soutenir l'affirmation classique : Vaut mieux prévenir que guérir auquel les intervenant(e)s en loisir auront à promouvoir pour écarter l'ampleur au mal du stress que le loisir thérapeutique résout par sa portée humaniste de type spirituel. Ils devront se redéfinir plus directement comme un service de la santé qui offre une programmation tenant compte des limites physiques et psychologiques de leur clientèle. L'environnement naturel et les équipements seront adaptés en vue de réduire le mal du stress en éludant ses causes premières pour aller de l'avant. Le loisir thérapeutique est la voie royale du devenir humain, où s'exprime l'identité profonde et véritable de la liberté d'être des personnes. Il constitue à la fois un environnement pour des actes de professionnalisme et l'exercice de l'action enjouée, c'est-à-dire sans chercher des résultats prédéterminés si ce n'est la quête de la santé mentale à visée optimale.

Les personnes vieillissantes et aînées ont ces capacités de créer leur expérience de loisir vers l'éveil d'un « état d'esprit; une façon d'être en paix avec soi-même et avec ce qu'on est en train de faire » (Neulinger, 1974, p. xvii) tout en agissant dans le domaine de leurs possibilités du risque de la décision et de l'action qui apporte un sens de la vie. Le loisir thérapeutique sera potentiellement porteur d'une véritable renaissance culturelle riche en significations des plus diverses. En fait, le temps consacré au loisir thérapeutique est un 
temps riche à l'éveil spirituel. Il évoque des sentiments et des émotions. Il représente également un retour aux sources, une paix et une connaissance intérieures, une activité de repos, une expérience de joie, une liberté totale, une fraternité et de l'amitié, un contact avec soimême et de ce que l'on ressent, une notion de plaisir, un sentiment d'évasion, un état d'âme et, en définitive, un antistress. Le loisir thérapeutique nécessite donc de vivre, de prendre son temps et d'être soi-même. Cette notion de santé liée au loisir thérapeutique encourage la convivialité, le dépassement, le plaisir et la critique sociale. Les personnes deviendront plus conscientes de leur potentiel vital et créateur puisqu'elles font l'expérience intime de la liberté d'être et sont alors plus responsables de leur rapport au temps et au monde par la pratique active de diverses expériences ludiques et sportives qui apportent le sentiment et l'émotion de mieux-être. Cette approche discursive du loisir thérapeutique suscite quelques questions d'appoints : (1) Le loisir thérapeutique contribue-t-il véritablement à l'élévation culturelle et morale des personnes vieillissantes et aînées?; (2) Le loisir thérapeutique sertil à rendre la vie humaine plus humaine?; et (3) Justifie-t-il une révélation et un lien durable avec la Transcendance?

\section{Enjeu du loisir \# 3 - L'éducation au loisir}

Le monde de l'éducation subit la fragmentation qui se caractérise par une perte du sens commun et de la crise de l'autorité (Arendt, 1972). De fait, l'école ne remplit plus la mission qu'elle prétend défendre dans son entièreté : « [d]es générations auront été formées selon des processus si disparates qu'on ne voit pas comment on pourra parler dans vingt ans d'une culture québécoise où se reconnaitraient des raisons communes » (Dumont, 1995, p. 157). L'échec relatif de la réforme scolaire est une preuve que la scolarisation a ses défaillances puisque l'on passe de réforme en réforme en oubliant que ce sont les jeunes qui devraient être la préoccupation ultime et non pas l'aspect technique et promotionnel d'un intellectualisme faisant ironiquement la gestion de « [1]'obsession des réformes [qui] nous a engagés dans un système d'éducation qui s'est bureaucratisée autour des réformes » (Rocher, 2010, p. 164). Du coup, ce que nous observons depuis les derniers 50 ans à partir de la révolution culturelle, c'est qu'au lieu de se libérer des entraves qu'elle prétend faire, l'éducation semble au contraire susciter plus de mal que de bien dans une perspective anomique des conflits de valeurs : « [1]'éducation est devenue banale, dans la tête des gens, parce qu'on en a évacué le sacré. C'est alors qu'on finit par cultiver l'ignorance. Les sommets de barbarie jusque-là impensables sont maintenant atteints au quotidien dans des comportements d'un égoïsme prodigieux » (Demers, 2012, p. 163). La société hypermoderne conditionne les jeunes faisant des expériences qui s'inscrivent dans un rythme si rapide qu'ils n'ont plus le temps d'aimer et de jouir du moment présent de la vie. Tout se fait dans l'instantané qui a son prix. Plus le temps d'apprécier la beauté. Tout se passe dans la laideur de l'ennui du quotidien véhiculant une perte de sens du temps et de l'éternité. La patience est quant à elle une sagesse oubliée et la science de l'opportunité une valeur méprisée. C'est pourquoi que « [1]'humanité en est venue à une période de son évolution, où une réflexion et une information sur ses fins dernières sont nécessaires à sa survie » (Fourastier, 1976, p. 205). ${ }^{11}$ Dans cette perspective, Morin (1999) affirme qu'il s'agit de comprendre l'unité de la personne dans la diversité; sa diversité dans l'unité du multiple, et en même temps, la multiplicité de l'Un. À cela, « la mission proprement spirituelle de l'éducation : enseigner la compréhension entre les personnes comme condition et garant de la solidarité intellectuelle et morale de l'humanité » (Morin, 1999, p. 51). La communauté de destin terrestre requiert donc de manière vitale la solidarité que l'éducation au loisir aura la fonction sociale de réguler puisque « [1] sage est patient et sait se taire jusqu'au moment 
opportun. Son secret est d'être accordé à la situation, d'être un homo tempestivus, [. . .] qui connaît l'heure juste pour intervenir à point. Il n'est ni homo faber, un homme travaillant toujours pressé pour qui le temps est de l'argent (time is money), ni homo ludens, qui dépense son temps à s'amuser sans assumer ses responsabilités » (Volant, 2003, p. 215). Le temps est un grand maître. Il s'agit de cultiver la patience afin d'apprécier des moments de réussite et de repos mérité.

Friedmann (1970) croit que l'éducation a un rôle qui dépasse la préparation à la vie professionnelle, sociale et personnelle. Pour lui, le rôle de l'éducation autorise une prise de conscience dans des voies de la sagesse malgré tous les résultats pernicieux et destructeurs de la puissance technoscientifique des sociétés de notre époque : "L'éducation véritable, dit-il, c'est l'homme transformant sa condition par des institutions rationnelles, mais aussi grâce à la transformation de soi par soi : c'est l'homme se prenant lui-même en main. L'éducation véritable, son succès ou son échec commande l'avenir de l'homme » (Friedmann, 1970, p. 442). De sorte que le temps bénéfique permet d'atteindre cette réalité éducationnelle laissant les pièces du puzzle reprendre sa mise en forme. Friedmann (1966) suggère également que l'éducation dans les écoles devrait se préoccuper à préparer les jeunes, non seulement au travail, mais également au loisir dans la projection éducative de l'ensemble des valeurs humanistes de type spirituel. Si l'éducation ne se réduit pas seulement à l'école proprement dite, elle pourrait garder cette capacité cruciale de remettre en cause certaines valeurs mortifères sur ses connaissances acquises quand Dumont (1981, p. 14) pose cette question ineffable : «Comment combattre des idéologies traditionnelles, comment abolir à la limite toute idéologie, sinon par l'éducation? ». Dans ce cas-là, l'action éducative devient un style de vie et favorise la régénérescence du tissu social par l'effet pondéré de la spiritualité du loisir.

Dans un contexte où plusieurs jeunes éprouvent des problèmes d'échecs scolaires et divers handicaps sociaux, la visée éthico-spirituelle de l'éducation au loisir sous-tend fermement que « l'individualité spirituelle de chacun devra être jalousement préservée pour le plus grand avantage de l'ensemble » (Rostand, 1953, p. 92), et ce, en vue de les raccrocher à l'éducation afin de favoriser leur participation pleine et entière à la vie sociale. L'éducation au loisir sera un temps à soi convivial et fructifiant dans des positionnements relationnels dont la responsabilisation des uns et des autres s'inscrit dans la vivacité de la vie (Salomé, 1999). Considérée comme un don Divin, l'éducation au loisir est un temps sacré qui permet de recentrer l'énergie vitale afin de pouvoir vaquer aux préoccupations quotidiennes.

La reconnaissance de la pédagogie de l'espoir s'avère cruciale dans la construction de l'identité chez l'enseigné(e). C'est pourquoi la notion d'espoir renferme en elle-même une dynamique d'avenir que les jeunes régénèrent au quotidien et qu'ils concrétisent dans diverses activités scolaires et parascolaires. On constate donc qu'il soit important de voir l'aspect positif des choses et non seulement leur côté sombre. Il existe inéluctablement chez les jeunes des forces insoupçonnées qui les légitiment de chercher et de trouver l'aspect positif des choses tout en gardant espoir en la vie. Pour leur parler d'avenir, il s'agira de leur montrer qu'il existe des solutions à les conscientiser aux problèmes de l'existence parce que l'espoir est une notion qui se communique et se transmet dans les actions de la vie quotidienne, si petites soient-elles. L'essentiel est que l'enseignant(e) démontre le plus de cohérence possible entre ses actions et son discours, mettant ainsi en oeuvre sa vision optimiste de l'existence. La pédagogie de l'espoir conduit à une réalité particulière, où l'on construit le climat éducatif sans autres règles et contraintes que celles qu'on se donne à travers le jeu du contrat implicite et de la relation pédagogique. Ceux-ci s'établissent entre l'enseignant(e) et l'enseigné(e) puisque le principe du jeu est a priori « une épreuve 
de soi » (Château, 1967, p. 61) qui permet de devenir, par la maîtrise personnelle, une meilleure personne. D'après Brouillette (2009), cette pédagogie de l'espoir aide à se fixer des buts, à se projeter dans l'avenir et à faire des plans tout en procurant un sentiment de sécurité et une conscientisation globale à l'échelle planétaire. Ainsi, " [i]l s'agit non de figurer le réel, mais de le transfigurer » (Fourastier, 1976, p. 273). La pédagogie de l'espoir est la recherche de prendre des décisions appropriées aux diverses situations en reconnaissant la visée éthico-spirituel de «vivre-bien avec et pour les autres dans des institutions justes et de s'estimer soi-même en tant que porteur de ce vœu » (Ricoeur, 1990, p. 406). L'éducation performative par la pédagogie de l'espoir légitime cette réalisation par les liens interactifs de la classe et par la recherche du sens commun de tous par l'entremise du réalisme optimiste.

Dans ces circonstances, une définition opératoire à l'éducation au loisir s'inspire des grandes lignes de la formulation suivante : «L'éducation au loisir peut être un processus qui veut permettre à [la personne] de se réaliser dans les différentes pratiques de loisir. Ce processus doit permettre l'acquisition de connaissances et doit favoriser la réalisation d'expériences susceptibles d'amener [la personne] à prendre conscience de son potentiel et de se prendre en charge dans ce domaine » (Conseil québécois de la jeunesse, des sports et de plein air, 1976, p. 9). En stimulant le développement d'une réflexion critique sur le monde, l'éducation au loisir permet aux personnes d'acquérir une meilleure maîtrise de soi face aux événements néfastes de la contemporanéité. L'approche préconisée de l'éducation au loisir n'est pas au service d'une société de la surperformance et de la surproductivité qui a une conception déficiente de l'enseigné(e) parce que l'« être humain est en perpétuel devenir; l'enfermer dans une définition, qu'elle soit formulée à l'école maternelle ou plus tard, c'est trahir sa liberté de devenir celui qu'il choisit d'être » (Jacquard, 2009, pp. 138-139). D'autant plus que l'esprit de compétition envers et contre tous est à proscrire parce que celui-ci mène régulièrement à des culs-de-sac qui annihilent l'esprit humain enfermé dans des situations limites : « à une époque dominée par le vide existentiel, la principale tâche de l'éducation, au lieu de se limiter à la transmission des traditions et du savoir, devrait consister à affiner en chacun l'aptitude à découvrir son orientation de sens » (Frankl, 2009, p. 65). Ainsi, l'éducation au loisir autorisera ce voyage intérieur de la quête de sens par la pédagogie de l'espoir qui justifie la recherche « en permanence de progresser, d'être meilleur que soi-même et non meilleur que les autres. [. . .] apprendre à rencontrer les autres pour se construire. [L'école] est au service de ceux qui s'adressent à elle pour qu'elle les aide à devenir eux-mêmes » (Jacquard, 2009, pp. 126, 128). Elle aura alors la tâche de répondre à des questions existentielles fondamentales : " Que sommes-nous? Qu'est-ce que l'homme? Que représente-t-il dans l'ensemble des choses? Qu'est-ce qu'une vie humaine? Qu'est-ce qui s'efface de l'univers [ou jaillit] quand périt un individu?» (Rostand, 1953, p. 19). L'humanisation de la jeunesse prend forme à travers la relation pédagogique, où la puissance performatrice des mots révèle toute son importance. Il s'avère essentiel de pratiquer ce syllogisme percutant afin de marteler l'esprit de l'enseigné(e) : «Si l'on vous dit que vous n'êtes pas bon, vous pourriez bien le croire. Le contraire est aussi vrai, car si l'on vous dit que vous êtes bon, vous pourriez bien le croire également. Alors, pourquoi risquer quoi que ce soit auprès des jeunes. Disons-leur qu'ils sont bons. Ils pourraient bien le croire et le devenir » (Demers, 2011, p. 146). Pour sortir les jeunes du vide spirituel et de la déshumanisation dont ils sont victimes, l'enseignant(e) prodiguera en transmettant ce réalisme optimiste. Pour ce faire, Demers (2008) propose l'établissement d'une programmation étoffée du sport et du loisir en milieu scolaire par une contribution effective des personnes assignées et compétentes. Cela signifie que les principaux décideurs du système scolaire ne devraient pas hésiter d'augmenter les heures en éducation physique dans le curriculum ainsi que l'octroi de fonds budgétaires nécessaires à la 
tenue de programmes intra-muraux et parascolaires (Roy, 2011). De même, les services techniques en loisir seront convoqués pour créer la synergie éducative de l'école par la responsabilisation. Être responsable signifie se porter garant d'une action ou répondre des actions que l'on pose. La responsabilité comporte un engagement moral et l'obligation de faire le nécessaire pour rendre les choses possibles. Pour que la responsabilité s'instaure, la personne reconnait dans sa réflexion les conséquences d'une initiative ou d'une tâche ainsi que le « retentissement affectif » (Ladrière, 1997, p. 148) de l'imputabilité personnelle que l'on nomme le sentiment de responsabilité. Lorsqu'une personne se responsabilise, sa spiritualité prend forme et donne un sens altruiste et convivial. Les jeunes qui ont une cause commune y découvriront cette raison de vivre en luttant envers ce qui les rend étrangers à eux-mêmes. Il s'agit de se brancher sur ce qui est essentiel à nos vies. Les difficultés et les obstacles que rencontrent les jeunes deviennent alors des éléments formateurs de cette quête intérieure du développement humain.

L'autoformation apparaît un concept-clé pour toutes les personnes qui ont le désir et la capacité de s'autoformer tous au long de leur vie, et ce, de façon permanente (Dumazedier, 2002). Il s'agit par l'entraînement mental de montrer aux jeunes cette pédagogie de l'espoir applicable à l'éducation au loisir et de leur montrer la distinction entre les différents temps sociaux de la vie quotidienne : le travail, la vie familiale, le loisir dans lequel il y a les engagements politiques, sociaux, spirituels/religieux constituant le temps existentiel à travers les parcours de vie. Il s'agit aussi d'apprendre à gérer l'accroissement du temps libre parce que « la société doit être préparée et éduquée pour le loisir » (Robinson \& Godbey, 2000, p. 315, trad.). L'autoformation est une voie performante pour développer l'apprentissage à la capacité et au désir de se former personnellement et collectivement dans la société en changement tout le long de la vie. Bien qu'elle réponde à des besoins spécifiques de société, l'école est un lieu intéressant de champ de pratique disciplinaire pour les intervenant(e)s en loisir qui sont des agent(e)s multiplicateurs pour récréer la vie sociale de l'école ainsi que de la salle de classe comme microcosme. Ils ont eux aussi la capacité de transmettre la pédagogie de l'espoir dans le réalisme optimiste parce que c'est dans la «mutualité que les gens acquièrent une valeur à la fois pour eux-mêmes et pour les autres; c'est dans l'échange, autrement dit, que se façonne la reconnaissance d'individus s'apparaissant comme partenaires et hommes capables » (Roberge, 2011, p. 53). Les intervenant(e)s en loisir ont donc un rôle important à jouer dans le milieu scolaire. Cela pose des questions pour un proche avenir : (1) Si l'école a pour mission de former au loisir, alors de quel type de loisir s'agit-il? (2) Comment pouvons-nous être de meilleurs agent(e)s multiplicateurs en enseignant le réalisme optimiste? (3) Est-ce que la pédagogie de l'espoir est transmissible aux jeunes qui n'ont plus rien à espérer d'éducateur(trice)s à l'esprit corporatiste excessif?

\section{Quelques mots aux fins de clore la discussion}

Dans les multiplicités des regards, nous avons donc réuni une série de tendances sociales par voie successive qui soulèvent plusieurs questions dont les réponses demeurent aléatoires et requièrent donc des temps de réflexion nécessairement disponibles pour un questionnement prospectif, où le lecteur a la responsabilité de construire son propre univers de sens du loisir. Ces tendances sociales permettent d'énoncer trois enjeux liés au loisir de la contemporanéité, soit l'action bénévole, le loisir thérapeutique et l'éducation au loisir. Ceux-ci sont des moments privilégiés au développement humain des potentialités et de l'atteinte des limites; la capacité d'exercer son pouvoir créateur en harmonie avec l'accomplissement personnel de ce que nous sommes comme personne vieillissante toujours en devenir par un humanisme de type spirituel. 
Ce processus favorise la fortification de la confiance en soi qui fait apparaître alors la possibilité d'une véritable renaissance culturelle laissant à la personne d'enrichir ses connaissances et d'aller à la rencontre des sociétés culturelles avoisinantes. Ces contextes de loisir sont progressivement un lieu de convivialité, d'amitié, de participation engagée, de solidarité et d'intégration sociale inclusive dans la communauté sociale. Les gestes gratuits deviennent palpables et s'y révèlent une manière étroite d'être dans le monde établissant un lien étroit avec le don et la générosité. Dès lors, au-delà de l'utilitarisme rationnel, les enjeux de l'action bénévole, du loisir thérapeutique et de l'éducation au loisir participent explicitement au déploiement d'un monde ré-enchanté. Dans cette vision dynamique de la promotion du loisir, il est alors possible qu'il devienne source de régénérescence du tissu social. Un défi responsable pour toute personne de bonne volonté est à notre portée, où la spiritualité du loisir détient un rôle fondamental au maintien des liens sociaux.

Le politique a l'obligation morale de se pencher sur ces réalités du loisir. En même temps, les personnes sont convoquées à donner, recevoir et rendre pour que s'instaure la libre circulation des biens et services dans la communauté sociale. Réorganiser la vie quotidienne de manière à rendre le temps libre plus révélateur et réduire le rythme effréné de la contemporanéité prendra le courage d'être ce que nous sommes dans la réalité des choses. Les intervenant(e)s en loisir sont convoqués à participer pour aider à définir l'éventualité d'un meilleur avenir. En ce sens, ces enjeux au loisir comme finalités ultimes se traduisent par l'affirmation de la vie tout simplement. Il est une manière d'être au monde liant une relation étroite à la fois avec la grâce, le don et la gratuité. Les pratiquants du loisir sont alors transportés à travers leurs spiritualités projetant un monde ludique qui dévoile ce qui est mystérieux et merveilleux à la fois. Au cœur de l'aventure humaine de chaque personne vieillissante, le loisir est associé aux notions d'authenticité et de liberté assurant à celui qui s'investit des moments privilégiés de calme menant au sentiment de repos et d'émotion de joie de vivre. Le loisir est alors le temps et le lieu, où se rencontrent d'authentiques moments d'espérance ici, maintenant et toujours.

Mon cœur est en repos, mon âme est en silence; le bruit lointain du monde expire en arrivant, comme un son éloigné Qu'affaiblit la distance, à l'oreille incertaine apportée par le vent. [...]

Dieu, pour le concevoir, a fait l'intelligence : Sous la nature enfin découvre son auteur! Une voix à l'esprit parle dans son silence : Qui n'a pas entendu cette voix dans son cœur?

- Alphonse de Lamartine (1790-1869)

Le vallon

\section{Remerciements}

J'aimerais souligner l'apport de M. Pierre Lalongé qui a fait une lecture critique de cet article et m'a apporté des conseils judicieux quant à la qualité de l'expression écrite. Qu'il en soit ici remercié.

\section{Notes}

1. Une distinction sémantique entre les concepts 'loisir' et 'loisirs' est ici suggérée. Le premier terme au singulier - le 'loisir' - fait référence au phénomène de la vie qu'est l'expérience du loisir. Cette acception au singulier représente la modalité d'existence du loisir comme 'état
De nouveau ce cortège muet

M'apportant leurs mots

L'avenir me réveille avec son silence

Je me joins au cortège

Une porte ouverte

Parle pour moi

De nouveau.

- William Stanley Merwin (1927-)

The Moving Target 
d'esprit' tandis que le deuxième terme au pluriel - les 'loisirs' - se définit comme un bien de consommation dans l'industrie de la culture de masse faisant référence aux multiples activités liées au monde de la [sur]consommation. Les 'loisirs' ici font l'objet de la gestion administrative de privatisation et de tarification dans la communauté locale (cf. Arendt, 1972; D'Amours, 1987).

2. On a qu'à imaginer l'époque de la Rome antique nourrissant sa population de Pain et des Jeux/Panem et Circenses dans un climat de violence; et que celle-ci se laisse berner à toutes sortes de caprices par des divertissements de natures fétichistes véhiculant notamment des valeurs épicuriennes.

3. La Déclaration universelle des droits de l'Homme stipule que « Toute personne a droit au repos et aux loisirs et notamment à une limitation raisonnable de la durée de travail et à des congés payés périodiques » (Déclaration universelle des droits de l'Homme, 1948, art. 24, http://www. un.org/fr/documents/udhr/).

4. L'acceptation de la notion anthropologique 'corps-âme-esprit' est un présupposé pour la compréhension des personnes quant à leurs dispositions éthico-spirituelles qui s'articulent autour $\mathrm{du}$ « respect et [de] l'obligation, structurés autour des besoins matériels, fourniss[ant] un point de départ pour une société plus juste et plus enracinée » (Holt, 2012, p. 83).

5. Dumazedier prétend que tout le débat sur le temps libre réside du point de vue même de l'observateur et sous l'angle du temps observé. Si les observations prennent comme point de référence la Révolution industrielle au dix-neuvième siècle, il est indéniable que les personnes de la contemporanéité profitent de plus de temps libre.

6. Dans cette même veine, Fourastier avance l'hypothèse des 40000 heures de travail considérant que les personnes des sociétés post-industrielles avec une technologie informatique ultraperformante et hautement mécanisées en viendront à la semaine de 30 heures avec 40 semaines de travail par année ce qui totalise 1200 heures annuellement. Cette vue de l'esprit exposé par cet auteur montre que les personnes continuerait à travailler jusqu'à 65 ans. Il s'agit de prévoir une durée de 35 à 40 ans de vie active. Si l'on multiplie 35 ans de travail par 1200 heures par an, cela donnerait 40000 heures de travail (cf. Laplante, 1966).

7. L'expression 'personnes vieillissantes' signifie à tous les âges de la vie des personnes avant l'âge de 65 ans et plus.

8. La définition de la notion de 'santé mentale' est la suivante : « la capacité de l'individu, du groupe et de l'environnement de réagir de manière à favoriser le bien-être personnel, le développement et l'utilisation des habilités mentales (cognitives, affectives et relationnelles), l'atteinte des buts individuels ou collectifs compatibles avec la justice, l'atteinte et la préservation des conditions d'égalité » (Raymaker \& Garrard, 2002, p. 22).

9. L'étymologie du terme bénévolat provient du latin 'benevolus' signifiant 'bonne volonté'.

10. L'action bénévole est un acte de participation sociale et de citoyenneté et influence, au plan local et associatif, nombre de services socioculturels, sportifs et religieux. Des bénévoles participent à la défense de l'environnement. Certains donnent de leur temps à la culture en contribuant à des groupes de chorales, de théâtres ou d'orchestres. Les municipalités font appel aux bénévoles qui donnent leur appui en cas de catastrophes naturelles ou pour combattre des incendies. Des volontaires participent aux activités à la bibliothèque ou à des activités sportives auprès de la jeunesse. Des personnes prennent sous tutelle des jeunes en détresse ou s'engagent dans les familles d'accueil. D'autres bénévoles s'impliquent dans des centres d'urgence pour soutenir les enfants maltraités, les victimes d'agression sexuelle ou s'investissent dans des activités parascolaires. Plusieurs accompagnent les personnes mourantes, les handicapés, les malades mentaux, les personnes aînées, les sans-abri et les pauvres.

11. La prospective morale de Fourastier le mena à prédire la semaine de travail à 30 heures et une vie active d'environ 35 ans. Il est aussi à l'origine de l'expression des 'Trente glorieuses' (1947-1973). L'auteur affirme que « [1] a religion de demain, c'est tout ce qui, dans la religion d'hier, est accordé à la science d'aujourd'hui. La vérité religieuse est la conscience la moins obscure, qu'à chaque âge de son histoire, l'humanité peut prendre d'elle-même et de l'univers » (1976, p. 272).

\section{Références}

Arendt, H. (1972). La crise de la culture: Huit exercices de pensée politique. Paris: Gallimard.

Baum, G. (1999). Nationalisme et mouvements sociaux contre l'hégémonie du marché. Le Devoir, Montréal, A9. 
Beaupré, S. (2012). L'objectivation de la pratique enseignante. Dans N. Bouchard \& M. Gagnon (dir.), L'Éthique et Culture religieuse en question: Réflexions critiques et prospectives (pp. 85-101). Québec: Presses de l'Université du Québec.

Bédard, M.-Ë., \& Carbonneau, H. (2012). L'apport d'une formation en loisir pour les intervenants et les propriétaires de résidences privées pour les personnes aînées: Quels en sont les effets? Vie et Vieillissement, 10(2), 42-49.

Béland, C., Bouchard, J.-É., \& Girard, J.-P. (2012). Un dialogue intergénérationnel sur le modèle coopératif. Montréal, QC: Fides.

Bellefleur, M. (1975-1976). Une certaine approche de l'idée de loisir. Loisir plus, 4(5-6), 16-17.

Bellefleur, M. (2002). Le loisir contemporain: essai de philosophie sociale. Sainte-Foy, QC: Presses de l'Université du Québec.

Berger, P., \& Luckmann, T. (1986). La construction sociale de la réalité. Paris: Méridiens Klincksieck.

Bouet, M. (1969). Les motivations des sportifs. Paris: Presses universitaires de France.

Bourdieu, P. (1980). Le sens pratique. Paris: Minuit.

Brault, M.-M.T. (1990). Le travail bénévole à la retraite. Québec, QC: Institut québécois de recherche sur la culture, 25 .

Brouillette, V. (2009). Comment parler d'avenir aux jeunes? Vie pédagogique, 154.

Caillé, A. (2007). Anthropologie du don. Paris: Desclée de Brouwer/La Découverte.

Charpentier, M., \& Quéniart, A., et coll. (2007). Pas de retraite pour l'engagement citoyen. Québec, QC: Presses de l'Université du Québec.

Château, J. (1967). Jeux de l'enfant. Dans R. Callois (dir.), Jeux et sports (pp. 49-149). Paris: Gallimard.

Conseil québécois de la jeunesse, des sports et de plein air. (1976). L'éducation au loisir au Québec: étude préliminaire. Document de travail.

D'Amours, M. (1987). La privatisation des loisirs municipaux. Cahiers d'études du loisir, 4, 1-47.

D'Amours, M., \& Pronovost, G. (1990). Les études du loisir: Pour une nouvelle lecture de la société. Loisir et Société/Society and Leisure, 13(1), 15-38.

Delisle, M.-A. (1987). La république du silence, solitude et vieillissement. Québec, QC: Laboratoire de recherches sociologiques de l'Université Laval.

Demers, P. (2008). Élever la conscience humaine par l'éducation. Québec, QC: Presses de l'Université du Québec.

Demers, P. (2011). L'humanité de l'obscurité à la lumière: L'éducation pour rendre le pouvoir à l'être humain. Québec, QC: Presses de l'Université du Québec.

Demers, P. (2012). Éduquer et vivre à partir du cour: Une vision renouvelée de la vie et de l'éducation? Québec, QC: Card.

Deschênes, G. (2002). Pour une spiritualité du loisir. Loisir et Société/Society and Leisure, 25(1), $173-202$.

Deschênes, G. (2007). Le loisir, Une quête de sens: Essai de théologie pratique. Québec, QC: Presses de l'Université Laval.

Deschênes, G. (2011). L'anthropologie spirituelle du loisir: l'homo faber-religiosus-ludens. Counseling et Spiritualité/Counselling and Spirituality, 30(2), 57-85.

Dumazedier, J. (1962). Vers une civilisation du loisir? Paris: Seuil.

Dumazedier, J. (1990a). Pour un renouveau de la recherche en sciences sociales du loisir. Loisir et Société, 13(1), 63-76.

Dumazedier, J. (1990b). Contributions des personnes âgées à la société du temps libre. Gérontologie et Société, 55, 20-31.

Dumazedier, J. (1993). Épilogue. Dans C. Attias-Donfut, G. Pronovost, \& N. Samuel (dir.), Temps libre et modernité: Mélange en l'honneur de Joffre Dumazedier (pp. 361-390). Sainte-Foy, QC: Presses de l'Université du Québec.

Dumazedier, J. (2002). Penser l'autoformation: Société d'aujourd'hui et pratiques d'autoformation. Lyon: Chronique sociale.

Dumont, F. (1981). Une révolution culturelle? Dans F. Dumont, J. Hamelin, \& J.-P. Montminy (dir.), Idéologies au Canada-Français 1940-1976 (pp. 5-31). Québec, QC: Presses de 1'Université Laval.

Dumont, F. (1995). Raisons communes. Montréal: Boréal.

Fahmy, M., et coll. (2012). Régénérations: Propositions citoyennes pour un Québec intergénérationnel. Montréal, QC: Fides. 
Fourastier, J. (1976). Le long chemin des hommes. Paris: Robert Laffont.

Frankl, V. (2009). Nos raisons de vivre: À l'école du sens de la vie. Paris: InterÉditions-Dunod. (L'original fut publié en 1969).

Friedmann, G. (1966). 7 études sur l'homme et sur la technique. Paris: Denoël/Gonthier.

Friedmann, G. (1970). La puissance de la sagesse. Paris: Gallimard.

Gagnon, É., \& Sévigny, A. (2000). Permanence et mutations du monde bénévole. Recherches sociographiques, 41(3), 529-544.

Genoe, R. (2010). Leisure as resistance within the context of dementia. Leisure Studies, 29(3), 303-320.

Godbey, G. (1997). Leisure and leisure services in the 21st century. State College, PA: Venture Publishing.

Godbout, J. T. (2007). Ce qui circule entre nous. Paris: Seuil.

Godbout, J. T., \& Caillé, A. (1992). L'esprit du don. Montmagny: Boréal.

Grafanaki, S., Pearson, D., Cini, F., Godula, D., McKenzie, B., Nason, S., \& Anderegg, M. (2005). Sources of renewal: A qualitative study on experience and role of leisure in the life of counsellors and psychologists. Counselling Psychology Quarterly, 18(1), 31-40.

Guillemard, A.-M. (2002). De la retraite mort sociale à la retraite solidaire: La Retraite une Mort Sociale (1972) revisitée trente ans après. Gérontologie et Société, 102, 53-66.

Hall, M., Knighton, T., Reed, P., Bussière, P., McRae, D., \& Bowen, P. (1998). Canadiens dévoués, Canadiens engagés: Points saillants de l'Enquête nationale de 1997 sur le don, le bénévolat et la participation. Cat. No: 71-542-XIE. Ottawa, ON: Ministère de l'Industrie.

Harvey, S. (2003). Stratégies et activités d'apprentissage chez les bénévoles en loisir au Québec. Loisir et Société/Society and Leisure, 26(2), 471-474.

Haworth, J., \& Lewis, S. (2005). Work, leisure and well-being. British Journal of Guidance \& Counselling, 33(1), 37-41.

Heintzman, P. (2008). Leisure-spiritual coping: A model for therapeutic recreation and leisure services. Therapeutic Recreation Journal, 42(1), 56-73.

Henderson, K. (1981). Motivations and perceptions of volunteerism as leisure activity. Journal of Leisure Research, 13(3), 208-218.

Holt, A. (2012). À la recherche du socialisme démocratique: La pensée politique de George Orwell et de Simone Weil. Esprit, 387.

Institut de la statistique du Québec. (2012). Vieillissement, réalités sociales, économiques et de santé. Récupérée de http://www.bdso.gouv.qc.ca/docs-ken/flex/ken_tbl_bord_0001/ tbl_bord_index.html

Jacquard, A. (2009). Le compte à rebours a-t-il commencé? Paris: Les documents Stock.

Karlis, G. (2004). Leisure and recreation in Canadian society: An introduction. Toronto, ON: Thompson Educational.

Kelly, J. R. (1987). Freedom to be: A new sociology of leisure. New York, NY: Macmillan.

Kelly, J. R. (2012). Leisure (4ème éd.). Urbana, IL: Sagamore.

Kelly, J. R., \& Freysinger, V. J. (2000). 21st century leisure: Current issues. College Station, PA: Venture.

Kraus, R. G. (2000). Leisure in a changing America. Needham Heights, MA: Allyn \& Bacon.

Lachance, J. (2011). L'adolescence hypermoderne: Le nouveau rapport au temps. Québec, QC: Presses de l'Université Laval.

Ladrière, J. (1997). L'éthique dans l'univers de la rationalité. Montréal, QC: Fides.

Lamari, M., \& Ménard, C. (2012). Démocratiser les loisirs et faciliter l'accès aux activités sportives: évaluation d'une initiative d'économie sociale visant les populations handicapées ou démunies au Québec, Canada. Leisure/Loisir, 36(1), 37-52.

Laplante, R. (1966). Les 40,000 heures. Paris: Action nationale. Récupérée de http://bibnum2.banq. qc.ca/bna/actionationale/src/1966/10/07/1966-10-07.pdf

Levy, P. (1995). Qu'est-ce que le virtuel? Paris: La Découverte.

Lipovetski, G. (1983). L'ère du vide, essais sur l'individualisme contemporain. Paris: Gallimard.

Lipovetski, G. (1987). L'empire de l'éphémère. Paris: Gallimard.

Lockstone-Binney, L., Holmes, K., Smith, K., \& Baum, T. (2010). Volunteers and volunteering in leisure: Social science perspective. Leisure Studies, 29(4), 435-455.

Loesch, L. C., \& Wheeler, P. T. (1982). Principles of leisure counseling. Minneapolis, MN: Educational Media. 
Lord, J., \& Hutchinson, P. (1979). Intégration sociale et loisirs. Islington, ON: Leisurability Publications.

Lord, J., \& Hutchinson, P. (2007). Pathways to inclusion: Building a new story with people and communities. Concord, ON: Captus Press.

Martin, A. R. (1975). Leisure and our inner resources. Park and Recreation, 10, 1a-16a.

Marx, K. (1996). Manuscrit de 1844. Paris: Flammarion (L'original fut publié en 1844).

Masson, C. (1991). Un mouvement ouvert. Colloque des animateurs scouts et guides du Québec, 1-8. Montréal: La Presse.

Morin, E. (1999). Les sept savoirs nécessaires à l'éducation du futur. Paris: Organisations des Nations unies pour l'éducation, la science et la culture.

Mounier, É. (2010). Le personnalisme. Paris: Presses universitaires de France. (L'original fut publié en 1949.)

Neulinger, J. (1974). The psychology of leisure. Springfield, MA: C. C. Thomas.

Pronovost, G. (1983). Temps, culture et société. Sainte-Foy, QC: Presses de l'Université du Québec.

Pronovost, G. (1993). Travaillent-ils trop?: Note au sujet de l'ouvrage de Juliet B. Shor. Loisir et Société/Society and Leisure, 16(1), 235-240.

Pronovost, G. (1997). Loisir et société: Traité de sociologie empirique. Québec, QC: Presses de l'Université du Québec.

Pronovost, G., \& Henri, P. (1996). Évolution de l'emploi du temps au Québec, 1986-1992. Québec, Ministère des Affaires municipales: Direction du loisir et des programmes à la jeunesse.

Raymaker, P., \& Garrard, N. (2002). Santé mentale et vieillissement. Ottawa, ON: Conseil consultatif national sur le troisième âge.

Ricard, F. (1992). La génération lyrique: Essai sur la vie et l'oeuvre des premiers-nés du baby-boom. Montréal, QC: Boréal.

Ricoeur, P. (1990). Soi-même comme un autre. Paris: Gallimard.

Rifkin, J. (1995). La fin du travail. Paris/Québec, QC: Découverte/Boréal.

Roberge, J. (2011). Du sens de la justice: Partage et pratique à l'aune d'une herméneutique critique. Dans A. Kerlan \& D. Simard (dir.), Paul Ricoeur et la question éducative (pp. 39-55). Québec, QC: Presses de 1'Université Laval.

Robichaud, S. (1998). Le bénévolat: Entre le coeur et la raison. Chicoutimi, QC: Jean-Claude Larouche.

Robinson, J. P. (1991). How Americans use their time. New York, NY: Praeger.

Robinson, J. P., \& Godbey, G. (2000). Time for life: The surprising ways Americans use their time. University Park, PA: The Pennsylvania State University.

Rocher, F. (2010). Guy Rocher: Entretiens. Montréal, QC: Boréal.

Rostand, J. (1953). Ce que je crois. Paris: Grasset.

Roussel, H., Ouellette, P., Singleton J., \& Hall, A.-M. (2012). Les tâches professionnelles et la satisfaction au travail des intervenants en loisir oeuvrant en milieu institutionnel québécois. Leisure/Loisir, 36(2), 211-229.

Roy, J. (2011). Quête identitaire et réussite scolaire: Une étude de cas - La pratique d'activités parascolaires dans le réseau collégiale. Québec, QC: Presses de l'Université du Québec.

Salomé, J. (1999). Le courage d'être soi: L'art de communiquer en conscience. Paris: Du Relié.

Schleifer, M., et coll. (2010). Valeurs et sentiments chez les adolescents: Comment avoir un dialogue qui favorise le respect mutuel? Québec: Presses de l'Université du Québec.

Schnapper, D. (1997). Contre la fin du travail. Paris: Textuel.

Schor, J. (1991). The overworked American: The unexpected decline of leisure. New York, NY: Basic Books.

Schumacher, E. F. (1978). Small is beautiful: Une société à la mesure de l'homme. Paris: Seuil.

Searle, M. S., \& Brayley, R. E. (2000). Leisure services in Canada. State College, PA: Venture.

Sobel, R. (1992). Working in America. Book review. Philadelphia Inquirer, February 23, K1.

Statistique Canada. (2001). Projections démographiques pour le Canada, les provinces et les territoires 2000-2026. Ottawa, ON: Ministre de l'Industrie.

Thibault, A., Albertus, P., \& Fortier, J. (2007). Rendre compte et soutenir l'action bénévole des jeunes. Ottawa, ON: Réseaux canadiens de recherche en politiques publiques. Récupérée de http://www.rcrpp.org

Thibault, A., \& Fortier, J. (2003). Comprendre et développer le bénévolat en loisir dans un univers technique et 'clientéliste'. Loisir et Société/Society and Leisure, 26(2), 315-344. 
Tocqueville, A. de. (1968). De la démocratie en Amérique. Paris: Gallimard (L'original fut publié en 1835-1840).

Toffler, A. (1980). La troisième vague. Paris: Denoël.

Veblen, T. (1899). Théorie de la classe de loisir. Paris: Gallimard.

Vézina, M., \& Crompton, S. (2012). Le bénévolat au Canada. Tendances sociales canadiennes. No 11-008 au catalogue. Ottawa, ON: Statistique Canada.

Volant, É. (1976). Le jeu des affranchis-Confrontation Marcuse-Moltmann. Montréal, QC: Fides.

Volant, É. (2003). La maison de l'éthique. Montréal, QC: Liber.

Weber, M. (1964). L'éthique protestante et l'esprit du capitaliste. Paris: Plon. (L'original fut publié en 1904-1905).

Weil, S. (1949). L'enracinement. Paris: Gallimard.

Wiesel, É. (1958). La nuit. Paris: Minuit.

Wunenburger, J.-J. (2003). L'imaginaire. Paris: Presses universitaires de France. 